\title{
KINETIC HIERARCHIES AND MACROSCOPIC LIMITS FOR CRYSTALLINE STEPS IN $1+1$ DIMENSIONS*
}

\author{
DIONISIOS MARGETIS ${ }^{\dagger}$ AND ATHANASIOS E. TZAVARAS A $^{\ddagger}$
}

\begin{abstract}
We apply methods of kinetic theory to study the passage from particle systems to nonlinear partial differential equations (PDEs) in the context of deterministic crystal surface relaxation. Starting with the near-equilibrium motion of $N$ line defects ("steps") with atomic size $a$, we derive coupled evolution equations ("kinetic hierarchies") for correlation functions $F_{n}^{a}$, which express correlations of $n$ consecutive steps. We investigate separately the evaporation-condensation and the surface diffusion dynamics in $1+1$ dimensions when each step interacts repulsively with its nearest neighbors. In the limit $a \rightarrow 0$ with $N a=\mathcal{O}(1)$, where $a$ is appropriately nondimensional, the first equations of the hierarchies reduce to known evolution laws for the surface slope profile. The remaining PDEs take the form of simple continuity equations, which we solve exactly and, thereby, connect continuous limits of $F_{n}^{a}$ with the slope profile. In addition, we construct a particular example of $F_{n}^{a}$ asymptotically for small but finite $a$ by regularization of measures. In the limit $a \rightarrow 0$, this construction yields singular correlation functions.
\end{abstract}

Key words. kinetic theory, epitaxial growth, crystal surface, Burton-Cabrera-Frank model, particle system, Bogoliubov-Born-Green-Kirkwood-Yvon hierarchy, closure, evaporation-condensation, surface diffusion, correlation function, step chemical potential, macroscopic limit, propagation of chaos

AMS subject classifications. 35Q99, 74A50, 74A60, 80M35, 82C22, 82C24, 82C70, 82D25

DOI. $10.1137 / 080726495$

1. Introduction. In the past several decades, significant mathematical advances have been witnessed in nonequilibrium statistical mechanics. An important research direction is to relate the dynamics of large particle systems to nonlinear partial differential equations (PDEs) for associated macroscopic variables. The linkage between particle systems and kinetic equations goes back to the works of Boltzmann and Grad for the Boltzmann equation (see, e.g., [4] for the derivation of the Boltzmann equation). This theme has been developed in various contexts describing deterministic or stochastic particle dynamics (see, e.g., Pulvirenti [30] and references therein).

A rich class of systems comprises crystalline materials. In particular, surfaces of crystals have received considerable attention because of their use in the fabrication of novel small devices, e.g., [9, 16, 24]. At sufficiently low temperatures, the evolution of crystal surfaces is driven by the lateral motion of line defects ("steps"), which can be perceived as particles in an appropriate sense. PDEs for the surface height have been formally derived from step motion, e.g., [23, 26, 28, 31].

In this article we apply methods of kinetic theory, in the spirit of [30], to describe the evolution of line defects at the nanoscale and its macroscopic descriptions. Each

*Received by the editors June 7, 2008; accepted for publication (in revised form) December 3, 2008; published electronically March 25, 2009.

http://www.siam.org/journals/mms/7-3/72649.html

$\dagger$ Department of Mathematics and Institute for Physical Science and Technology and Maryland NanoCenter, University of Maryland, College Park, MD 20742-4015 (dio@math.umd.edu). This author's research was supported in part by NSF-MRSEC DMR0520471 at the University of Maryland.

${ }^{\ddagger}$ Department of Mathematics, University of Maryland, College Park, MD 20742-4015 (tzavaras@ math.umd.edu); and Department of Applied Mathematics and Institute for Applied and Computational Mathematics (FORTH), University of Crete, GR 71409 Heraklion, Greece (tzavaras@tem. uoc.gr). This author's research was partially supported by the National Science Foundation and the program "Pythagoras" of the Greek Secretariat for Research.

1428 
step is allowed to interact only with its nearest neighbors. Coupled evolution equations ("kinetic hierarchies") are derived for appropriately defined functions expressing step correlations. Such hierarchies offer an alternative to the discrete schemes for steps. Correlation functions convey additional information relative to the step density (or surface slope) captured by macroscopic PDEs of thermodynamic origin. Our objective is to connect these hierarchies with the macroscopic limit, where the continuous surface height is the dependent variable. We also investigate analytically the behavior of step correlations for small yet finite step height, or a finite number of steps, which might conceivably offer a description of the system at an intermediate ("mesoscopic") scale.

Our work is motivated by the broader issue of making systematic predictions for step correlations possibly measured in experiments. This task requires a theory that is more elaborate than the usual macroscopic PDEs for the height variable. Models of step-position correlations are not well developed. The present article aims to make a contribution in this direction. (For implications of step-position distributions, see, e.g., [1] and references therein.)

To place the present work in the proper context, note that there are three major theoretical approaches to epitaxy. One approach relies on kinetic Monte Carlo simulations for the motion of classical atoms on a lattice, e.g., [6, 27], and lies beyond the scope of the present article. A second approach $[13,14,16]$ resorts to discrete simulations via models for line defects of the type of the Burton, Cabrera, and Frank (BCF) model [2]. A third approach relies on macroscopic laws such as PDEs for the surface height $[23,26,28,31,33,35]$. This perspective is well suited for largescale predictions but becomes questionable near facets [5, 22] and in the presence of surface instabilities such as step bunching [10]. Macroscopic laws usually stem from thermodynamics and mass conservation [33, 35]. Alternatively, such laws can be derived from discrete schemes for steps when the step density varies sufficiently slowly in space $[19,26,28,31]$. Formally, the macroscopic limit is approached by taking $a / \lambda \rightarrow 0$ with fixed surface slope, where $a$ is the step height and $\lambda$ is a macroscopic length.

The physics of steps has been described mainly near thermodynamic equilibrium. Steps move by mass conservation [2] following various transport processes, most notably surface diffusion and evaporation-condensation. In surface diffusion, adsorbed atoms ("adatoms") diffuse on terraces while atoms attach or detach at step edges; this transport process is dominant for sufficiently small structures. In evaporationcondensation, atoms are exchanged between steps and the surrounding vapor. We study separately the dynamics of each process and restrict attention to $1+1$ dimensions. Our formulation accounts for step repulsions including entropic [11] and elastic dipole $[18,25,32]$ step interactions.

The analysis is developed for three distinct models: a toy model (TM) which serves as a simple paradigm; an evaporation-condensation model (ECM); and a surface diffusion model (SDM) for steps. Given a discrete particle system describing the motion of line defects (steps), we introduce measure-valued functions: the empirical measure $F_{1}^{a}$ and higher-order correlation functions $F_{n}^{a}$. These functions are defined in terms of the usual delta function and measure the density of steps $(n=1)$ or higherorder correlations $(n>1)$. They turn out to satisfy coupled, nonlocal evolution equations, similar to the Bogoliubov, Born, Green, Kirkwood, and Yvon (BBGKY) hierarchies of statistical mechanics [12, 34]; see (3.15), (3.19), and (3.25). The particle systems describing step evolution are characterized by short-range interactions (in contrast to the mean field interaction of the McKean model used in [30]). This feature is reflected to the form of the hierarchies and accompanying properties; for instance, 
$F_{n}^{a}$ in (3.19) does not admit a closure relation in the form of propagation of chaos, i.e., the product $F_{n}^{a}=F_{n-1}^{a} F_{1}^{a}$, when $a$ is finite.

The novel element in this work is the description of step motion through the machinery of kinetic hierarchies and the emergence of thermodynamics-based PDEs for the height as macroscopic limits of such hierarchies in $1+1$ dimensions. The discrete schemes offer a Lagrangian description of the step train. The kinetic hierarchies provide a Eulerian description instead. The correlation functions $F_{n}^{a}$ for $n \geq 2$ convey information beyond the step density or surface slope, $F_{1}^{a}$; hence, they may conceivably transcend the single PDE for the surface height and provide a mesoscopic picture linking nanoscale step motion with the macroscopic PDE.

In the macroscopic limit, $a \rightarrow 0$ and $N \rightarrow \infty$ with $N a=\mathcal{O}(1)$, where $N$ is the number of steps and $a$ is appropriately nondimensional, we show that each hierarchy reduces to a set of decoupled transport equations; see (4.4) with (5.1)-(5.2). The equations for the macroscopic step density $F_{1}$ coincide with the usual parabolic PDEs for the surface height. The equations for the macroscopic correlation functions $F_{n}$, $n \geq 2$, have the form of transport equations and can be solved explicitly. Their solution leads to expressions that propagate the correlation functions of the initial data and include the propagation of chaos as a special case.

Finally, for small but finite $a$, we follow a somewhat different perspective and explore the character of $F_{n}^{a}$ as measures. By regularizing these measures via mollifiers, we construct a particular example of $F_{n}^{a}$ that can solve the hierarchies asymptotically; see our main Proposition 6.3. This construction yields $F_{n}^{a}$ that become singular as $a \rightarrow 0$. The issue of what initial data might lead to these particular $F_{n}^{a}$ needs to be further explored.

It should be noted that our starting models have certain limitations. First, they are restricted to $(1+1)$-dimensional surface mounds: steps are straight and form a monotonic train; i.e., we assume that $\partial_{x} h<0$ throughout where $h(x, t)$ is the continuous surface height, $x$ is the space coordinate, and $t$ is time. The steps are ordered initially and, because of their noncrossing condition, are assumed to retain this ordering for $t>0$. The initial data and governing equations for the step positions are deterministic; thus, stochastic effects are neglected. Each step is assumed to interact only with its nearest neighbors [14]. Our formulation focuses on surfaces that relax to become flat (in the absence of material deposition from above). The case with material deposition can easily be included in the kinetic description, although deposition leads to very different PDEs at the macroscale [29]. The starting models rely on nearequilibrium concepts, making use of ideas that permeate the BCF theory [2]; farfrom-equilibrium processes $[3,21]$ are not touched upon.

The present kinetic description admits several extensions not included here. The effect of bulk stress, by which steps interact beyond their nearest neighbors [7, 17], is amenable to similar but more elaborate analysis. Stochastic effects coming from randomized initial data can be treated in this context but lie beyond our present scope. We focus on straight steps; evolution in $2+1$ dimensions, although tractable within our framework, is not addressed here.

The remainder of this article is organized as follows. In section 2 we review the discrete schemes and their macroscopic limits: in section 2.1 we introduce a sufficiently general step velocity law; in section 2.2 we outline concepts relevant to the macroscopic limit of stepped surfaces; in section 2.3 we describe a simple scheme (TM) to clarify the techniques involved; in section 2.4 we focus on evaporation-condensation dynamics (ECM); and in section 2.5 we turn our attention to surface diffusion dynamics (SDM). In section 3 we introduce the kinetic description: in section 3.1 we 


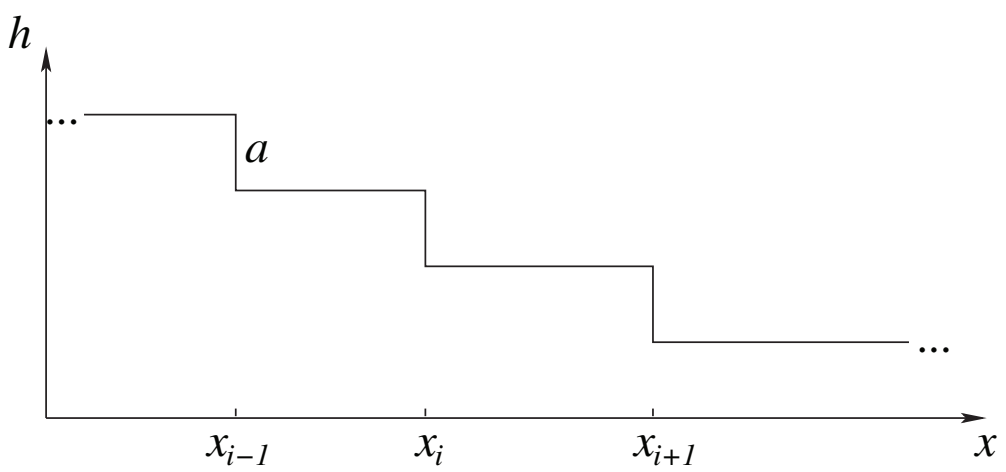

FIG. 2.1. Monotonic step train in $1+1$ dimensions; $x_{i}(t)$ is the position of the ith step.

define step correlation functions $F_{n}^{a}$; and in section 3.2 we derive BBGKY-type hierarchies for $F_{n}^{a}$. In section 4 we study the kinetic hierarchies in the macroscopic limit. In section 5 we derive sufficiently general solutions of the macroscopic laws for step correlation functions. In section 6 we provide an example of a construction for $F_{n}^{a}$, asymptotically for small $a$. Finally, in section 7 we summarize our results and discuss limitations of our analysis. For notational economy, we suppress the explicit dependence on time $(t)$ unless we indicate otherwise.

2. Discrete schemes for step evolution and their macroscopic limits. In this section we review discrete schemes describing the evolution of steps in $1+1$ dimensions and the corresponding macroscopic limits. We focus on surface relaxation (when surfaces evolve to become planar), assuming that no external sources are present. The discrete schemes describe the step velocity as a function of step positions. Details on the assumptions and derivations can be found elsewhere, e.g., in [16, 23, 24].

2.1. Discrete schemes and step velocities. The physical setting consists of $N$ repulsively interacting steps of height $a$ at positions $x=x_{i}(t), x_{i+1}>x_{i}$, where $i$ is the step number $(i=1, \ldots, N$ and $N \gg 1)$. In this low-temperature picture, $N$ remains fixed: the number of steps is set by the miscut angle of the crystal surface in an experiment or via screw periodic boundary conditions in theory [8]. The step train is monotonic with steps of the same orientation, say, steps descending in the direction of increasing $x$; see Figure 2.1.

Throughout the analysis we use $x_{i}$ and $a$ as nondimensional variables and parameter, respectively, by taking the macroscopic length $\lambda$ of the surface to be unity. ${ }^{1}$ It follows that the (discrete) surface height is piecewise constant, being equal to $h_{i}$ for $x_{i}<x<x_{i+1}$ where

$$
h_{i}-h_{i+1}=a .
$$

We assume that each step interacts only with its nearest neighbors [13, 14]. The velocity of the $i$ th step reads

$$
\dot{x}_{i}=\zeta_{i}\left(x_{i-2}, x_{i-1}, x_{i}, x_{i+1}, x_{i+2} ; a\right),
$$

\footnotetext{
${ }^{1}$ Alternatively, define $\tilde{x}_{i}=x_{i} / \lambda$ and $\tilde{a}:=a / \lambda$ and then drop the tildes so that $x_{i}$ and $a$ become nondimensional. The time $t$ and the equations for the step velocities are also properly nondimensionalized.
} 
where the dot denotes differentiation with respect to time $(t)$ and $\zeta_{i}$ are given functions which depend on the microscopic parameter $a$. This form is sufficiently general to encompass evaporation-condensation and surface diffusion processes. In evaporationcondensation, the step velocity is driven by the step chemical potential, i.e., the change in the step free energy caused by the addition or removal of atoms at the step. Then, $\zeta_{i}$ depends only on $x_{i}$ and $x_{i \pm 1}$ and (2.2) is a discrete version of the fully continuum model formulated by Spohn [35]; see section 2.4. In surface diffusion, the step velocity is determined by the adatom fluxes incident upon the step edge from adjacent terraces; each flux depends on the difference of chemical potentials at steps bounding the corresponding terrace [14]. Then, $\zeta_{i}$ couples $x_{i}$ with two of its neighbors on each side; see section 2.5 .

In the following, we set $\zeta_{i}=\zeta$ which simplifies the analysis yet leaves almost intact the essential physics of step motion. We alert the reader that the order of variables in the function $\zeta$ matters: $\zeta$ exhibits algebraic singularities at regions where two of its consecutive arguments coincide [11, 15, 18, 25, 32].

2.2. Macroscopic limits: General framework. The macroscopic limit of (2.2) is meaningful if the terrace widths $x_{i+1}-x_{i}$ vary sufficiently slowly over the size of a sample containing a large number $N$ of steps. Formally, this limit is attained by taking $N \rightarrow \infty$ and $a \rightarrow 0$ with $N a=\mathcal{O}(1)$ so that the total change of height remains bounded. It is assumed that the step density,

$$
m_{i}=\frac{a}{x_{i+1}-x_{i}}
$$

remains $\mathcal{O}(1)$ and that the sequence $m_{i}$ approaches the positive surface slope $m(x)=$ $-\partial_{x} h$, with the macroscopic surface height $h(x, t)$ being sufficiently differentiable [23]. Accordingly, in the limit $a \rightarrow 0$ we have

$$
m_{i} \rightarrow m(x)=\left|\partial_{x} h(x)\right|, \quad x=x_{i} .
$$

In the same vein, $h_{i}$ approaches $h(x)$ with $h\left(x_{i}\right) \equiv h_{i}$; cf. (2.1).

Alternatively, (2.3) can result systematically from the constraint (2.1). If the sequence $x_{i}$ interpolates a continuous function, $x_{i}=X(s)$ where $s=i \delta$ and $\delta=\mathcal{O}(a)$, then $x_{i \pm 1}$ can be expanded in the Taylor series in $\delta$ as [10]:

$$
x_{i \pm 1}=X(s \pm \delta)=X(s) \pm \delta X_{s}(s)+\frac{\delta^{2}}{2} X_{s s}+\cdots, \quad \delta X_{s} \ll X .
$$

By reconciling (2.4) with (2.1) where $h_{i} \equiv h\left(x_{i}\right)$, we obtain $\delta X_{s}=a m(x, t)^{-1}$, which implies (2.3). The parameter $\delta$ signifies the small scale and can be eliminated from the resulting equations of motion; equivalently, set $\delta=a$ so that $s$ becomes the height variable $h$ and (2.4) reads as an expansion in powers of $a$.

Consequently, the step velocity $\dot{x}_{i}$ approaches a continuous function. Recall that the $i$ th terrace, $x_{i}<x<x_{i+1}$, is a level set for the surface height. Hence, $h\left(x_{i}, t\right)=$ constant, uniformly with $t$. Differentiation of this formula with respect to $t$ yields [23]

$$
\dot{x}_{i} \rightarrow \frac{\partial_{t} h(x)}{\left|\partial_{x} h(x)\right|} \quad\left(x=x_{i}\right) \quad w k,
$$

where the symbol " $w k$ " indicates convergence in the weak sense. It is then observed that upon using (2.1) we have

$$
a \sum_{i} \dot{x}_{i} \phi_{i} \rightarrow \int \mathrm{d} h \frac{\partial_{t} h(x)}{\left|\partial_{x} h\right|} \phi(x)=\int \mathrm{d} x \partial_{t} h \phi(x)
$$

for reasonably arbitrary $\phi_{i}$ and test function $\phi$, and fixed time $t$. 
2.3. Toy model. By choosing $\zeta=a^{-1}\left[g\left(x_{i}\right)-g\left(x_{i+1}\right)\right]$, we reduce $(2.2)$ to the simple difference scheme

$$
\dot{x}_{i}=\frac{1}{a}\left[g\left(x_{i}\right)-g\left(x_{i+1}\right)\right]
$$

where $g(x)$ is a differentiable and monotonically increasing function which may depend explicitly on $t$. From the viewpoint of surface motion, this law is not physical since it forces each step to "feel" only its one-side neighbor. This model will be used as a simple example to illustrate the forthcoming methods.

In the macroscopic limit, $a \rightarrow 0$ and $x_{i+1}-x_{i} \rightarrow 0$ with $m_{i}=\mathcal{O}(1)$, the right-hand side of (2.7) approaches the values

$$
\frac{g\left(x_{i}\right)-g\left(x_{i+1}\right)}{a}=-\frac{x_{i+1}-x_{i}}{a} \frac{g\left(x_{i+1}\right)-g\left(x_{i}\right)}{x_{i+1}-x_{i}} \rightarrow-\frac{\partial_{x} g(x)}{\left|\partial_{x} h(x)\right|} \quad w k ;
$$

recall that $\partial_{x} h<0$ and, thus, $h(x)$ is invertible. In view of (2.5), formula (2.7) reduces to the first-order PDE

$$
\partial_{t} h=-\partial_{x} g
$$

2.4. Evaporation-condensation model. The step velocity is now proportional to the difference of the chemical potential $\mu_{\mathrm{v}}$ of the surrounding vapor and the step chemical potential $\mu_{i}: \dot{x}_{i}=\kappa_{i}\left(\mu_{\mathrm{v}}-\mu_{i}\right)$ where $\kappa_{i}$ is a suitable mobility $\left(\kappa_{i}>0\right)$. We take $\kappa_{i} \equiv 1$ and $\mu_{\mathrm{v}} \equiv 0 .^{2}$ The chemical potential $\mu_{i}$ is a function of the adjacent terrace widths [14], and the velocity law (2.2) becomes

$$
\dot{x}_{i}=-\mu_{i}=\frac{g\left(w_{i-1}\right)-g\left(w_{i}\right)}{a}, \quad w_{i}:=m_{i}^{-1}, \quad m_{i}=\frac{a}{x_{i+1}-x_{i}},
$$

where $g(w)$ is differentiable for $w \neq 0$ and $\lim _{w \rightarrow+\infty} g(w)=0$; in particular, $g(w)=$ $A / w^{3}$ with $A>0$ for entropic or elastic-dipole interactions [11, 14, 18, 25, 32]. Equation (2.10) also states that $\mu_{i}$ stems from interactions of the $i$ th step with its nearest neighbors; viz., $\mu_{i}$ is defined by $[14,16]$

$$
\mu_{i}=\frac{\mathrm{d}}{\mathrm{d} x_{i}}\left[V\left(w_{i}\right)+V\left(w_{i-1}\right)\right],
$$

where $V(w)$ is the pair interaction energy; thus, $g(w)=-V^{\prime}(w)$.

The use of (2.4) in (2.10) yields the macroscopic step chemical potential

$$
\mu_{i} \rightarrow \mu(x)=\frac{1}{m(x)} \partial_{x} g\left(\frac{1}{m}\right)=-\partial_{h} g\left(\frac{1}{m} \circ x(h)\right) \quad w k
$$

where $m(x, t)=-\partial_{x} h(x, t)$ is the slope and $x(h)$ is the inverse of $h(x)$. Thus, (2.10) reduces to the second-order $\mathrm{PDE}$

$$
\partial_{t} h=-\partial_{x} g\left(\frac{1}{m}\right) .
$$

This PDE is consistent with Spohn's model for evaporation-condensation [35]; see also [36] for an application of (2.13) to the grooving of grain boundaries.

\footnotetext{
${ }^{2}$ In principle, the material mobility is dimensional. The substitution used here is justified via absorption of any dimensional constant into $\mu_{i}$ and the time $t$.
}

Copyright $@$ by SIAM. Unauthorized reproduction of this article is prohibited. 
The chemical potential $\mu$ can be defined variationally in a way consistent with continuum thermodynamics [23]. Define the total step interaction energy $E^{\text {int }}$ by

$$
E^{\mathrm{int}}=\sum_{i} V\left(w_{i}\right) \sim \frac{1}{a} \int \mathrm{d} x m(x) V(w) \quad\left(w=m^{-1}\right)
$$

where the symbol " " here means that $a E^{\text {int }}-\int \mathrm{d} h V(w) \rightarrow 0$ as $a \rightarrow 0$. By definition of $\mu_{i}$, the variation of $E^{\text {int }}$ is ${ }^{3}$

$$
\dot{E}^{\mathrm{int}}=\sum_{i} \frac{\partial E^{\mathrm{int}}}{\partial x_{i}} \frac{\mathrm{d} x_{i}}{\mathrm{~d} t}=\sum_{i} \mu_{i} v_{i} \sim \frac{1}{a} \int \mathrm{d} x \mu(x) \partial_{t} h .
$$

This limit is computed from (2.14) via integration by parts with vanishing boundary terms:

$$
a \dot{E}^{\mathrm{int}} \rightarrow \int \mathrm{d} x \partial_{m}(m V) \partial_{t} m=\int \mathrm{d} x \partial_{x} \partial_{m}(m V) \partial_{t} h, \quad m=-\partial_{x} h .
$$

The comparison of (2.16) to (2.15) furnishes (2.12) weakly provided that $g(w) \equiv$ $m^{2} \partial_{m} V(w)=-V^{\prime}(w)$, where $w=m^{-1}$.

2.5. Surface diffusion model. In this case, the step velocity equals the difference of mass fluxes incident upon the step edge from adjacent terraces:

$$
\dot{x}_{i}=a^{-1}\left[J_{i-1}\left(x_{i}\right)-J_{i}\left(x_{i}\right)\right],
$$

where $J_{i}(x)$ is the (nondimensional) mass flux on the $i$ th terrace $\left(x_{i}<x<x_{i+1}\right)$. This $J_{i}$ accounts for atom attachment-detachment at step edges and is proportional to the difference of step chemical potentials $[14,16]$, e.g.,

$$
J_{i}\left(x_{i}\right)=-\frac{1}{a} \frac{\mu_{i+1}-\mu_{i}}{q+x_{i+1}-x_{i}},
$$

where $q$ is a kinetic length expressing the relative importance of two processes, namely, diffusion of adatoms on terraces and attachment-detachment of atoms at step edges.

In view of (2.18) and (2.10), the step velocity law (2.17) reads [14]

$$
\dot{x}_{i}=\frac{1}{a^{3}}\left[\frac{g\left(w_{i+1}\right)-2 g\left(w_{i}\right)+g\left(w_{i-1}\right)}{q+x_{i+1}-x_{i}}-\frac{g\left(w_{i}\right)-2 g\left(w_{i-1}\right)+g\left(w_{i-2}\right)}{q+x_{i}-x_{i-1}}\right] .
$$

This law can be simplified in extreme kinetic regimes. When terrace diffusion is the slowest process, we have $q \ll x_{i+1}-x_{i}[20]$. By contrast, when attachment-detachment is the slowest process, we take $q \gg x_{i+1}-x_{i}$ [20]. This latter kinetic regime is often referred to as "attachment-detachment limited" (ADL) kinetics and characterizes a wide class of materials and conditions [16]. By restricting attention to ADL kinetics, we reduce $(2.19)$ to

$$
\dot{x}_{i}=\frac{g\left(w_{i+1}\right)-3 g\left(w_{i}\right)+3 g\left(w_{i-1}\right)-g\left(w_{i-1}\right)}{a^{3}},
$$

setting $q=1$ or properly absorbing $q$ in $g$ and time $(t)$ without loss of generality.

\footnotetext{
${ }^{3}$ This definition applies only to monotonic step trains in $1+1$ dimensions. In $2+1$ dimensions, $E^{\text {int }}$ must be replaced by the total step energy which should include a step curvature (self-energy) contribution [23].
} 
In the macroscopic limit, the right-hand side of (2.17) becomes

$$
\frac{J_{i-1}-J_{i}}{a} \rightarrow-\frac{1}{m} \partial_{x} J(x)=\partial_{h} J(x(h)) \quad w k,
$$

where $h=h(x)$ and, by virtue of (2.12) and (2.18), the macroscopic flux $J(x)$ is given by

$$
J_{i}\left(x_{i}\right) \rightarrow J(x)=-\frac{1}{m} \partial_{x} \mu=-\frac{1}{m} \partial_{x}\left[\frac{1}{m} \partial_{x} g\left(m^{-1}\right)\right]=-\partial_{h}^{2} g\left(m^{-1} \circ x(h)\right) \quad w k .
$$

Thus, (2.20) reduces to the fourth-order PDE

$$
\partial_{t} h=\partial_{x}\left\{m^{-1} \partial_{x}\left[m^{-1} \partial_{x} g\left(m^{-1}\right)\right]\right\}=-m \partial_{h}^{3} g\left(m^{-1} \circ x(h)\right) .
$$

The more general step model $(2.19)$ can be treated similarly. In this case, the large-scale adatom flux is $J(x)=-[1+\tilde{q} m(x)]^{-1} \partial_{x} \mu$, where $\tilde{q}$ is the limit of $q / a$ as $a \rightarrow 0$. The macroscopic PDE for the height $h$ reads

$$
\partial_{t} h=-m \partial_{h}\left[(1+\tilde{q} m)^{-1} m \partial_{h}^{2} g\left(m^{-1} \circ x(h)\right)\right] .
$$

3. Kinetic description of step evolution. The objectives of this work are to obtain a kinetic description of the step evolution laws in the finite- $a$ range and study the macroscopic limit $a \rightarrow 0$ from this perspective. In this section we formulate the kinetic hierarchies for finite $a$. The description of particle systems via kinetic functions is commonplace in the derivation of the Boltzmann equation [4] and has been employed in the literature to describe the evolution of particle systems [30] and their macroscopic limits.

3.1. Correlation functions. We define the measure-valued functions

$$
\begin{aligned}
F_{1}^{a}\left(y_{1}\right) & :=a \sum_{i=1}^{N} \delta\left(y_{1}-x_{i}\right), \\
F_{n}^{a}(\vec{y}) & :=a \sum_{i=1}^{N} \prod_{j=1}^{n} \delta\left(y_{j}-x_{i+j-1}\right)=a \sum_{i} \delta\left(\vec{y}-\vec{x}_{(i)}\right),
\end{aligned}
$$

where

$$
\vec{y}:=\left(y_{1}, y_{2}, \ldots, y_{n}\right), \quad \vec{x}_{(i)}:=\left(x_{i}, x_{i+1}, \ldots, x_{i+n-1}\right),
$$

the time dependence of $x_{k}$ 's is suppressed, and the prefactor $a$ (or $N^{-1}$ since $N a=$ $\mathcal{O}(1))$ in $F_{n}^{a}$ is included to associate $F_{1}^{a}$ with the surface height; cf. (3.4) below. It should be borne in mind that $F_{n}^{a}$ depend nontrivially on $a$ via the $x_{k}$ 's and velocity law (2.2). Each $F_{n}^{a}$ plays the role of an $n$-step correlation function.

Evidently, we have

$$
\int_{\mathbb{R}} \mathrm{d} y_{n} F_{n}^{a}(\vec{y})=F_{n-1}^{a}\left(\vec{y}^{n}\right), \quad n \geq 2,
$$

where $\mathbb{R}$ is the set of real numbers and $\vec{y}^{p}$ denotes the $(n-1)$-dimensional vector stemming from $\vec{y}$ by omission of its $p$ th component, e.g., $\vec{y}^{n}:=\left(y_{1}, y_{2}, \ldots, y_{n-1}\right)$. A 
translation invariant form of $F_{n}^{a}$ for $n=2$ has been used previously in a study of step bunching [17]. The empirical measure ("singlet density") $F_{1}^{a}(x)$ satisfies

$$
\int_{x_{a}}^{x_{b}} \mathrm{~d} x F_{1}^{a}(x)=\left(N_{b}-N_{a}\right) a,
$$

where $N_{b}-N_{a}$ is the number of steps between $x=x_{a}$ and $x=x_{b}$; thus, (3.4) represents the absolute value of the surface height change in $\left(x_{a}, x_{b}\right)$.

Note that by (3.4) the singlet density $F_{1}^{a}$ approaches the positive surface slope $m$ in the weak sense

$$
F_{1}^{a}(x) \rightarrow F_{1}(x):=m(x)=-\partial_{x} h \quad w k,
$$

which means that

$$
\int \mathrm{d} x F_{1}^{a}(x) \phi(x) \rightarrow \int \mathrm{d} x m(x) \phi(x) \quad \text { as } a \rightarrow 0 .
$$

3.2. BBGKY-type hierarchies. In this section we derive evolution equations for the $n$-step correlation functions $F_{n}^{a}$ defined in (3.1) by using the general model (2.2). The resulting hierarchy is then simplified for specific choices of models, namely, the TM (simple difference scheme), the ECM, and the SDM introduced in section 2.

First, we focus on the singlet density $F_{1}^{a}$. The differentiation of (3.1) with respect to $t$ and use of (2.2) with $\zeta_{i}=\zeta$ yield

$$
\begin{aligned}
& \partial_{t} F_{1}^{a}(x)=-\sum_{i} \dot{x}_{i} \delta^{\prime}\left(x-x_{i}\right) \\
& =-\partial_{x} \sum_{i} \zeta\left(x_{i-2}, x_{i-1}, x, x_{i+1}, x_{i+2} ; a\right) \delta\left(x-x_{i}\right) \\
& =-\partial_{x}\left[\int_{\mathbb{R}^{4}} \mathrm{~d} u \mathrm{~d} w \mathrm{~d} y \mathrm{~d} z \sum_{i} \zeta(u, w, x, y, z ; a) \delta\left(u-x_{i-2}\right) \delta\left(w-x_{i-1}\right)\right. \\
& \left.\times \delta\left(x-x_{i}\right) \delta\left(y-x_{i+1}\right) \delta\left(z-x_{i+2}\right)\right] .
\end{aligned}
$$

Relations of this type are addressed rigorously in the proof of Proposition 4.1. It follows that

$$
\partial_{t} F_{1}^{a}(x)+\partial_{x}\left[\int_{\mathbb{R}^{4}} \mathrm{~d} u \mathrm{~d} w \mathrm{~d} y \mathrm{~d} z \zeta(u, w, x, y, z ; a) F_{5}^{a}(u, w, x, y, z)\right]=0 .
$$

This is the first equation of the kinetic hierarchy.

The remaining equations of the hierarchy for $F_{n}^{a}(n \geq 2)$ are derived similarly. The hierarchy generalizes for $n \geq 5$ because the step motion law in principle couples five steps. Thus, we have to distinguish the cases $n=2,3,4$ and $n \geq 5$. For $n=2$ we have

$$
\begin{aligned}
\partial_{t} F_{2}^{a}(x, y) & +\partial_{x}\left[\int_{\mathbb{R}^{3}} \mathrm{~d} z \mathrm{~d} w \mathrm{~d} u \zeta(u, w, x, y, z ; a) F_{5}^{a}(u, w, x, y, z)\right] \\
& +\partial_{y}\left[\int_{\mathbb{R}^{3}} \mathrm{~d} z \mathrm{~d} w \mathrm{~d} u \zeta(w, x, y, z, u ; a) F_{5}^{a}(w, x, y, z, u)\right]=0 .
\end{aligned}
$$

Copyright $@$ by SIAM. Unauthorized reproduction of this article is prohibited. 
In the same vein, $F_{3}^{a}$ satisfies

$$
\begin{aligned}
\partial_{t} F_{3}^{a}(x, y, z) & +\partial_{x}\left[\int_{\mathbb{R}^{2}} \mathrm{~d} w \mathrm{~d} u \zeta(u, w, x, y, z ; a) F_{5}^{a}(u, w, x, y, z)\right] \\
& +\partial_{y}\left[\int_{\mathbb{R}^{2}} \mathrm{~d} w \mathrm{~d} u \zeta(w, x, y, z, u ; a) F_{5}^{a}(w, x, y, z, u)\right] \\
& +\partial_{z}\left[\int_{\mathbb{R}^{2}} \mathrm{~d} w \mathrm{~d} u \zeta(x, y, z, u, w ; a) F_{5}^{a}(x, y, z, u, w)\right]=0 .
\end{aligned}
$$

Note that each of $F_{1}^{a}, F_{2}^{a}$, and $F_{3}^{a}$ is coupled only with $F_{5}^{a}$.

For $n=4$ the form of the evolution equation is different. We obtain

$$
\begin{aligned}
\partial_{t} F_{4}^{a}(x, y, z, u) & +\partial_{x}\left[\int_{\mathbb{R}^{2}} \mathrm{~d} v \mathrm{~d} w \zeta(v, w, x, y, z ; a) F_{6}^{a}(v, w, x, y, z, u)\right] \\
& +\partial_{y}\left[\int_{\mathbb{R}} \mathrm{d} v \zeta(v, x, y, z, u ; a) F_{5}^{a}(v, x, y, z, u)\right] \\
& +\partial_{z}\left[\int_{\mathbb{R}} \mathrm{d} v \zeta(x, y, z, u, v ; a) F_{5}^{a}(x, y, z, u, v)\right] \\
& +\partial_{u}\left[\int_{\mathbb{R}^{2}} \mathrm{~d} v \mathrm{~d} w \zeta(y, z, u, v, w ; a) F_{6}^{a}(x, y, z, u, v, w)\right]=0 .
\end{aligned}
$$

The evolution equations for $n \geq 5$ are cast to the form

$$
\begin{aligned}
\partial_{t} F_{n}^{a}(\vec{y}) & +\partial_{y_{1}}\left[\int_{\mathbb{R}^{2}} \mathrm{~d} p \mathrm{~d} s \zeta\left(p, s, y_{1}, y_{2}, y_{3} ; a\right) F_{n+2}^{a}(p, s, \vec{y})\right] \\
& +\partial_{y_{2}}\left[\int_{\mathbb{R}} \mathrm{d} s \zeta\left(s, y_{1}, y_{2}, y_{3}, y_{4} ; a\right) F_{n+1}^{a}(s, \vec{y})\right] \\
& +\sum_{j=3}^{n-2} \partial_{y_{j}}\left[\zeta\left(y_{j-2}, y_{j-1}, y_{j}, y_{j+1}, y_{j+2} ; a\right) F_{n}^{a}(\vec{y})\right] \\
& +\partial_{y_{n-1}}\left[\int_{\mathbb{R}} \mathrm{d} s \zeta\left(y_{j-3}, y_{j-2}, y_{j-1}, y_{j}, s ; a\right) F_{n+1}^{a}(\vec{y}, s)\right] \\
& +\partial_{y_{n}}\left[\int_{\mathbb{R}^{2}} \mathrm{~d} s \mathrm{~d} p \zeta\left(y_{j-2}, y_{j-1}, y_{j}, s, p ; a\right) F_{n+2}^{a}(\vec{y}, s, p)\right]=0 .
\end{aligned}
$$

Equations (3.8)-(3.12) are equivalent to the step velocity law (2.2). Therefore, we have replaced the coupled ordinary differential equations for the Lagrangian coordinates $x_{i}(t)$ by a set of coupled PDEs for the Eulerian variables $F_{n}^{a}(\vec{y})$. The advantage of this formulation lies in the ease by which one can connect the singlet density $F_{1}^{a}$ (or surface slope $m$ ) to step correlations.

Second, the derived PDEs for $F_{n}^{a}$ transcend the original definition (3.1) of $F_{n}^{a}$ in terms of delta functions: the PDEs admit both smooth and nonclassical solutions. Such solutions can express the behavior of step correlations at different scales, from the nanoscale to the macroscale, for a corresponding variety of initial data that can be very different from delta functions. ${ }^{4}$

\footnotetext{
${ }^{4}$ We alert the reader that the same system of evolution laws holds if the $F_{n}^{a}$ 's describe joint probability density functions for step positions under (2.2) with random initial data for $x_{i}$.
}

Copyright $@$ by SIAM. Unauthorized reproduction of this article is prohibited. 
3.3. Simple difference scheme (TM). In light of (2.7), the function $\zeta$ is

$$
\zeta(u, w, x, y, z ; a) \equiv a^{-1}[g(x)-g(y)]
$$

which in turn leads to considerable simplifications of evolution laws (3.8)-(3.12). Specifically, evolution equations (3.9)-(3.12) for $F_{n}^{a}$ with $n \geq 2$ are recast to a unified form.

The transport equations for $F_{n}^{a}$ now read as follows:

$$
\begin{aligned}
n=1: \quad \partial_{t} F_{1}^{a}(x) & +\partial_{x}\left\{a^{-1} \int \mathrm{d} y[g(x)-g(y)] F_{2}^{a}(x, y)\right\}=0, \\
n \geq 2: \quad \partial_{t} F_{n}^{a}(\vec{y}) & +\sum_{j=1}^{n-1} \partial_{y_{j}}\left\{a^{-1}\left[g\left(y_{j}\right)-g\left(y_{j+1}\right)\right] F_{n}^{a}(\vec{y})\right\} \\
& +\partial_{y_{n}}\left\{\int \mathrm{d} p a^{-1}\left[g\left(y_{n}\right)-g(p)\right] F_{n+1}^{a}(\vec{y}, p)\right\}=0,
\end{aligned}
$$

where indication of the integration region (here, $\mathbb{R}$ ) is omitted. Note that each $F_{n}^{a}$ is explicitly coupled only with $F_{n+1}^{a}$ where the equations of motion for $n \geq 2$ contain local terms linear in $F_{n}^{a}$.

3.4. Evaporation-condensation case (ECM). In this case, we have recourse to the step velocity law (2.10). Notice that, in comparison to the TM (2.7), $\dot{x}_{i}$ now explicitly depends on $x_{i+1}-x_{i}$ and $x_{i}-x_{i-1}$, i.e., the widths of adjacent terraces. The function $\zeta$ of $(2.2)$ reads

$$
\zeta(u, w, x, y, z ; a) \equiv \frac{1}{a}\left[g\left(\frac{x-w}{a}\right)-g\left(\frac{y-x}{a}\right)\right] .
$$

The hierarchy for $F_{n}^{a}$ from (3.8)-(3.12) is described by

$$
\begin{array}{r}
n=1: \quad \partial_{t} F_{1}^{a}(x)+\partial_{x}\left\{\int \mathrm{d} z \mathrm{~d} y \frac{1}{a}\left[g\left(\frac{x-z}{a}\right)-g\left(\frac{y-x}{a}\right)\right] F_{3}^{a}(z, x, y)\right\}=0, \\
n=2: \quad \partial_{t} F_{2}^{a}(x, y)+\partial_{x}\left\{\int \mathrm{d} z \frac{1}{a}\left[g\left(\frac{x-z}{a}\right)-g\left(\frac{y-x}{a}\right)\right] F_{3}^{a}(z, x, y)\right\} \\
+\partial_{y}\left\{\int \mathrm{d} z \frac{1}{a}\left[g\left(\frac{y-x}{a}\right)-g\left(\frac{z-y}{a}\right)\right] F_{3}^{a}(x, y, z)\right\}=0, \\
\begin{array}{r}
n \geq 3: \quad \partial_{t} F_{n}^{a}(\vec{y})+\partial_{y_{1}}\left\{\int \mathrm{d} p \frac{1}{a}\left[g\left(\frac{y_{1}-p}{a}\right)-g\left(\frac{y_{2}-y_{1}}{a}\right)\right] F_{n+1}^{a}(p, \vec{y})\right\} \\
+\sum_{j=2}^{n-1} \partial_{y_{j}}\left\{\frac{1}{a}\left[g\left(\frac{y_{j}-y_{j-1}}{a}\right)-g\left(\frac{y_{j+1}-y_{j}}{a}\right)\right] F_{n}^{a}(\vec{y})\right\} \\
+\partial_{y_{n}}\left\{\int \mathrm{d} p \frac{1}{a}\left[g\left(\frac{y_{n}-y_{n-1}}{a}\right)-g\left(\frac{p-y_{n}}{a}\right)\right] F_{n+1}^{a}(\vec{y}, p)\right\}=0 .
\end{array}
\end{array}
$$


We add a few comments on (3.17)-(3.19). First, evolution equation (3.17) is coupled directly only to $F_{3}^{a}$ since each step $i$ interacts with its nearest neighbors. However, steps acting as elastic dipoles interact in pairs even across many terraces. Such extended interactions, although expected to leave the macroscopic limit for $F_{1}^{a}$ essentially unchanged [23,33], should couple directly $F_{1}^{a}$ with $F_{n}^{a}$ for $n \geq 3$. Similar modifications are expected to occur in the other equations.

We note that the product form $F_{n}^{a}=F_{n-1}^{a} F_{1}^{a}$ does not offer a closure for the present hierarchy. This can be verified by direct substitution in (3.17)-(3.19).

3.5. Surface diffusion case (SDM). The case with surface diffusion deserves special attention because of its dominance in transport processes with surface features of sufficiently small size. With recourse to (2.20), we now set

$$
\zeta(u, w, x, y, z ; a) \equiv \frac{1}{a}\left[g\left(\frac{z-y}{a}\right)-3 g\left(\frac{y-x}{a}\right)+3 g\left(\frac{x-w}{a}\right)-g\left(\frac{w-u}{a}\right)\right]
$$

in the general hierarchy (3.8)-(3.12). The velocity of each step is now explicitly coupled with the positions of two neighbors on each side.

The evolution equations for $F_{n}^{a}$ generalize for $n \geq 5$. The hierarchy reads

$$
\begin{aligned}
& \partial_{t} F_{1}^{a}(x)+\partial_{x}\{ \int \frac{1}{a^{3}}\left[g\left(\frac{z-y}{a}\right)-3 g\left(\frac{y-x}{a}\right)+3 g\left(\frac{x-w}{a}\right)-g\left(\frac{w-u}{a}\right)\right] \\
& \times\left.F_{5}^{a}(u, w, x, y, z) \mathrm{d} y \mathrm{~d} z \mathrm{~d} w \mathrm{~d} u\right\}=0 \quad(n=1), \\
& \partial_{t} F_{2}^{a}(x, y)+\partial_{x}\left\{\int \frac{1}{a^{3}}\left[g\left(\frac{z-y}{a}\right)-3 g\left(\frac{y-x}{a}\right)+3 g\left(\frac{x-w}{a}\right)-g\left(\frac{w-u}{a}\right)\right]\right. \\
&\left.\times F_{5}^{a}(u, w, x, y, z) \mathrm{d} z \mathrm{~d} w \mathrm{~d} u\right\} \\
&+\partial_{y}\left\{\int \frac{1}{a^{3}}\left[g\left(\frac{u-z}{a}\right)-3 g\left(\frac{z-y}{a}\right)+3 g\left(\frac{y-x}{a}\right)-g\left(\frac{x-w}{a}\right)\right]\right.
\end{aligned}
$$

$$
\left.\times F_{5}^{a}(w, x, y, z, u) \mathrm{d} z \mathrm{~d} w \mathrm{~d} u\right\}=0 \quad(n=2),
$$

$$
\begin{aligned}
\partial_{t} F_{3}^{a}(x, y, z)+\partial_{x}\left\{\int \frac{1}{a^{3}}\left[g\left(\frac{z-y}{a}\right)-3 g\left(\frac{y-x}{a}\right)+3 g\left(\frac{x-w}{a}\right)-g\left(\frac{w-u}{a}\right)\right]\right. \\
\left.\times F_{5}^{a}(u, w, x, y, z) \mathrm{d} u \mathrm{~d} w\right\} \\
+\partial_{y}\left\{\int \frac{1}{a^{3}}\left[g\left(\frac{u-z}{a}\right)-3 g\left(\frac{z-y}{a}\right)+3 g\left(\frac{y-x}{a}\right)-g\left(\frac{x-w}{a}\right)\right]\right. \\
\left.\times F_{5}^{a}(w, x, y, z, u) \mathrm{d} u \mathrm{~d} w\right\} \\
+\partial_{z}\left\{\int \frac{1}{a^{3}}\left[g\left(\frac{w-u}{a}\right)-3 g\left(\frac{u-z}{a}\right)+3 g\left(\frac{z-y}{a}\right)-g\left(\frac{y-x}{a}\right)\right]\right.
\end{aligned}
$$

$$
\left.\times F_{5}^{a}(x, y, z, u, w) \mathrm{d} u \mathrm{~d} w\right\}=0 \quad(n=3),
$$

Copyright (c) by SIAM. Unauthorized reproduction of this article is prohibited. 


$$
\begin{aligned}
& \partial_{t} F_{4}^{a}(x, y, z, u)+\partial_{x}\left\{\int \frac{1}{a^{3}}\left[g\left(\frac{z-y}{a}\right)-3 g\left(\frac{y-x}{a}\right)+3 g\left(\frac{x-s}{a}\right)-g\left(\frac{s-w}{a}\right)\right]\right. \\
& \left.\times F_{6}^{a}(w, s, x, y, z, u) \mathrm{d} w \mathrm{~d} s\right\} \\
& +\partial_{y}\left\{\int \frac{1}{a^{3}}\left[g\left(\frac{u-z}{a}\right)-3 g\left(\frac{z-y}{a}\right)+3 g\left(\frac{y-x}{a}\right)-g\left(\frac{x-w}{a}\right)\right]\right. \\
& \left.\times F_{5}^{a}(w, x, y, z, u) \mathrm{d} w\right\} \\
& +\partial_{z}\left\{\int \frac{1}{a^{3}}\left[g\left(\frac{w-u}{a}\right)-3 g\left(\frac{u-z}{a}\right)+3 g\left(\frac{z-y}{a}\right)-g\left(\frac{y-x}{a}\right)\right]\right. \\
& \left.\times F_{5}^{a}(x, y, z, u, w) \mathrm{d} w\right\} \\
& +\partial_{u}\left\{\int \frac{1}{a^{3}}\left[g\left(\frac{s-w}{a}\right)-3 g\left(\frac{w-u}{a}\right)+3 g\left(\frac{u-z}{a}\right)-g\left(\frac{z-y}{a}\right)\right]\right. \\
& \left.\times F_{6}^{a}(x, y, z, u, w, s) \mathrm{d} s \mathrm{~d} w\right\}=0 \quad(n=4), \\
& \partial_{t} F_{n}^{a}(\vec{y})+\partial_{y_{1}}\left\{\int \frac{1}{a^{3}}\left[g\left(\frac{y_{3}-y_{2}}{a}\right)-3 g\left(\frac{y_{2}-y_{1}}{a}\right)+3 g\left(\frac{y_{1}-s}{a}\right)-g\left(\frac{s-p}{a}\right)\right]\right. \\
& \left.\times F_{n+2}^{a}(p, s, \vec{y}) \mathrm{d} p \mathrm{~d} s\right\} \\
& +\partial_{y_{2}}\left\{\int \frac{1}{a^{3}}\left[g\left(\frac{y_{4}-y_{3}}{a}\right)-3 g\left(\frac{y_{3}-y_{2}}{a}\right)+3 g\left(\frac{y_{2}-y_{1}}{a}\right)-g\left(\frac{y_{1}-s}{a}\right)\right]\right. \\
& \left.\times F_{n+1}^{a}(s, \vec{y}) \mathrm{d} s\right\} \\
& +\sum_{j=3}^{n-2} \partial_{y_{j}}\left\{\frac { 1 } { a ^ { 3 } } \left[g\left(\frac{y_{j+2}-y_{j+1}}{a}\right)-3 g\left(\frac{y_{j+1}-y_{j}}{a}\right)+3 g\left(\frac{y_{j}-y_{j-1}}{a}\right)\right.\right. \\
& \left.\left.-g\left(\frac{y_{j-1}-y_{j-2}}{a}\right)\right] F_{n}^{a}(\vec{y})\right\} \\
& +\partial_{y_{n-1}}\left\{\int \frac { 1 } { a ^ { 3 } } \left[g\left(\frac{s-y_{n}}{a}\right)-3 g\left(\frac{y_{n}-y_{n-1}}{a}\right)+3 g\left(\frac{y_{n-1}-y_{n-2}}{a}\right)\right.\right. \\
& \left.\left.-g\left(\frac{y_{n-2}-y_{n-3}}{a}\right)\right] F_{n+1}^{a}(\vec{y}, s) \mathrm{d} s\right\} \\
& +\partial_{y_{n}}\left\{\int \frac { 1 } { a ^ { 3 } } \left[g\left(\frac{s-p}{a}\right)-3 g\left(\frac{p-y_{n}}{a}\right)+3 g\left(\frac{y_{n}-y_{n-1}}{a}\right)\right.\right. \\
& \left.\left.-g\left(\frac{y_{n-1}-y_{n-2}}{a}\right)\right] F_{n+2}^{a}(\vec{y}, p, s) \mathrm{d} s \mathrm{~d} p\right\}=0 \quad(n \geq 5) .
\end{aligned}
$$

The surface diffusion model described here allows for extensions similar to those mentioned in the context of the ECM. For example, incorporating long-range effects in the form of step interactions beyond nearest neighbors will modify considerably laws (3.21)-(3.25) but leaves intact (except for a renormalized material parameter) the macroscopic limit of $F_{1}^{a}[23,33]$. This aspect is not studied here.

Copyright (c) by SIAM. Unauthorized reproduction of this article is prohibited. 
4. Hierarchies in macroscopic limit. In this section we study the passage from kinetic hierarchies (3.8)-(3.12) to nonlinear PDEs for the three models of section 2. A key element is the singlet density $F_{1}^{a}$, which approaches the positive surface slope $m=\left|\partial_{x} h\right|$ as discussed in section 2 .

Because of the noncrossing condition for steps and the ordering of their positions (i.e., $x_{i}<x_{i+1}$ ), admissible $F_{n}^{a}$ are assumed to obey

$$
F_{n}^{a}\left(y_{1}, \ldots, y_{j}, \ldots, y_{k}, \ldots, y_{n}\right) \equiv 0 \quad \text { if } y_{j} \geq y_{k} \quad(j<k) .
$$

This restriction must be imposed on the initial data (say, at $t=0$ ) for $F_{n}^{a}$. We, henceforth, assume that this condition is preserved by the flow (for $t>0$ ).

4.1. The macroscopic limit of step evolution laws. In the macroscopic limit $a \rightarrow 0$ and $N \rightarrow \infty$ with $a N=\mathcal{O}(1)$, the correlation function $F_{n}^{a}$ defined in (3.1) converges weakly (along a subsequence) to a limit $F_{n}(\vec{y}, t)$ for each fixed $t$. In other words, we have

$$
\int \mathrm{d} \vec{y} \phi(\vec{y}, t) F_{n}^{a}(\vec{y}, t) \rightarrow \int \mathrm{d} \vec{y} \phi(\vec{y}, t) F_{n}(\vec{y}, t)
$$

for fixed $t$ and any smooth test function $\phi(\vec{y}, t)$.

We proceed to compute the equation that $F_{n}$ satisfies. We prove a proposition describing the macroscopic limit at the level of the scaled dynamics induced by (2.2).

Proposition 4.1. Suppose that the discrete scheme (2.2) satisfies the uniform limit

$$
\zeta_{i}^{a}:=\zeta_{i}\left(x_{i-2}, x_{i-1}, x_{i}, x_{i+1}, x_{i+2} ; a\right)=v\left(x_{i}\right)+o(1) \quad \text { as } a \rightarrow 0
$$

for some smooth velocity function $v(x)$, where $o(1) \rightarrow 0$ uniformly in $i$ as $a \rightarrow 0$. Then, $F_{n}(\vec{y}, t)$ satisfies the weak form of the equation

$$
\partial_{t} F_{n}(\vec{y})+\sum_{j=1}^{n} \partial_{y_{j}}\left[v\left(y_{j}\right) F_{n}(\vec{y})\right]=0, \quad \vec{y} \in \mathbb{R}^{n}, t>0 .
$$

The limit velocity of form (4.3) is attained in all three models of section 2 as $a \rightarrow 0$ and $N \rightarrow \infty$ with $N a=\mathcal{O}(1)$. Thus, once (2.5) or (4.3) is established, then the hierarchy reduces to (4.4) as $a \rightarrow 0$. This limit is discussed case by case in section 4.3: a proof of (4.3) is provided for surface diffusion dynamics in section 4.3.3; the proofs for the other models are similar.

Equation (4.4) is of the form

$$
\partial_{t} F_{n}+\operatorname{div}\left[\vec{v}(\vec{y}) F_{n}\right]=0,
$$

where $\vec{v}(\vec{y})=\left(v\left(y_{1}\right), \ldots, v\left(y_{n}\right)\right)$ and $\operatorname{div} \vec{f}:=\sum_{j=1}^{n} \partial_{y_{j}} f_{j}$ with $\vec{f}=\left(f_{1}, \ldots, f_{n}\right)$. In particular, for $n=1$, (4.4) becomes the PDE for the surface slope. Conservation law (4.4) resembles the usual continuity equation for fluids, which in the present context states that no steps are destroyed or created. This meaning appears natural if the surface is viewed as a continuum and $F_{n}$ is the joint probability density of $n$ particles; thus, (4.4) expresses conservation of the probability density $F_{n}$ with the associated flux $\overrightarrow{\mathcal{J}}_{n}=\vec{v} F_{n}$. 
Proof. For the discrete scheme (2.2), upon formally differentiating (3.1), we obtain

$$
\begin{aligned}
\partial_{t} F_{n}^{a} & =-\sum_{j=1}^{n} a \sum_{i=1}^{N} \delta\left(y_{1}-x_{i}\right) \ldots \delta^{\prime}\left(y_{j}-x_{i+j-1}\right) \ldots \delta\left(y_{n}-x_{i+n-1}\right) \dot{x}_{i+j-1} \\
& =-\sum_{j=1}^{n} \partial_{y_{j}}\left[a \sum_{i=1}^{N} \delta\left(y_{1}-x_{i}\right) \ldots \delta\left(y_{j}-x_{i+j-1}\right) \ldots \delta\left(y_{n}-x_{i+n-1}\right) \zeta_{i+j-1}^{a}\right] .
\end{aligned}
$$

A rigorous derivation of (4.6) is obtained as follows. The correlation function $F_{n}^{a}$ is properly defined, by the action of the measure $F_{n}^{a}$ on continuous test functions $\phi(\vec{y}, t)$ periodic on the variable $\vec{y}$, via the relation

$$
\int \mathrm{d} \vec{y} F_{n}^{a}(\vec{y}, t) \phi(\vec{y}, t)=a \sum_{i=1}^{N} \phi\left(x_{i}(t), \ldots, x_{i+n-1}(t), t\right) .
$$

If $\phi(\vec{y}, t)$ is a $C^{\infty}$ test function, periodic in $\vec{y}$ and compactly supported on $(0, T)$ in the $t$-variable, we employ the trivial identity

$$
\int_{0}^{T} \frac{\mathrm{d}}{\mathrm{d} t} \sum_{i=1}^{N} \phi\left(x_{i}(t), \ldots, x_{i+n-1}(t), t\right) \mathrm{d} t=0
$$

to derive

$$
\begin{aligned}
& \iint \mathrm{d} t \mathrm{~d} \vec{y} F_{n}^{a}(\vec{y}, t) \partial_{t} \phi(\vec{y}, t)=\int \mathrm{d} t a \sum_{i=1}^{N} \partial_{t} \phi\left(x_{i}(t), \ldots, x_{i+n-1}(t), t\right) \\
& =-\int \mathrm{d} t a \sum_{j=1}^{n} \sum_{i=1}^{N}\left[\partial_{y_{j}} \phi\left(x_{i}(t), \ldots, x_{i+n-1}(t), t\right)\right] \zeta_{i+j-1}^{a} \\
& =-\iint \mathrm{d} t \mathrm{~d} \vec{y} \sum_{j=1}^{n}\left[\partial_{y_{j}} \phi(\vec{y}, t)\right]\left[a \sum_{i=1}^{N} \delta\left(y_{1}-x_{i}\right) \ldots \delta\left(y_{n}-x_{i+n-1}\right) \zeta_{i+j-1}^{a}\right],
\end{aligned}
$$

which is precisely (4.6).

Next, we pass to the limit $a \rightarrow 0$ of the right-hand side in (4.7) by using

$$
\begin{aligned}
& \int \mathrm{d} \vec{y}\left[\partial_{y_{j}} \phi(\vec{y}, t)\right]\left[a \sum_{i=1}^{N} \delta\left(y_{1}-x_{i}\right) \ldots \delta\left(y_{n}-x_{i+n-1}\right) \zeta_{i+j-1}^{a}\right] \\
& =\int \mathrm{d} \vec{y}\left[\partial_{y_{j}} \phi(\vec{y}, t)\right] F_{n}^{a}(\vec{y}, t)\left[v\left(y_{j}\right)+o(1)\right] \\
& \rightarrow \int \mathrm{d} \vec{y}\left[\partial_{y_{j}} \phi(\vec{y}, t)\right] v\left(y_{j}\right) F_{n}(\vec{y}, t)
\end{aligned}
$$

which follows from (4.2) and (4.3). Thus, we obtain (4.4) as the equation describing the limiting dynamics.

4.2. Macroscopic limits of specific models. In this subsection we revisit the limits of section 2 in light of the kinetic hierarchies of section 3. Our goal is to derive limiting relations involving nonlocal terms of these hierarchies.

Copyright $@$ by SIAM. Unauthorized reproduction of this article is prohibited. 
4.2.1. Toy model. The differentiation of both sides of PDE (2.9) with respect to $x$ yields

$$
\partial_{t} m-\partial_{x}^{2} g(x)=0
$$

By identifying $m$ with the singlet density $F_{1}$, we conclude that (4.8) should stem from (3.14), which is the first equation in the hierarchy for $F_{n}^{a}$. Thus, we write

$$
\lim _{a \rightarrow 0} \int \mathrm{d} y \frac{1}{a}[g(x)-g(y)] F_{2}^{a}(x, y)=-\partial_{x} g \quad w k .
$$

More precisely, relation (4.9) is expressed by ${ }^{5}$

$$
\begin{aligned}
& \lim _{a \rightarrow 0} \int \mathrm{d} x \phi(x) \int \mathrm{d} y \frac{1}{a}[g(x)-g(y)] F_{2}^{a}(x, y) \\
& =-\lim _{a \rightarrow 0} \sum_{i} \phi\left(x_{i}\right)\left[g\left(x_{i+1}\right)-g\left(x_{i}\right)\right] \\
& =-\lim _{a \rightarrow 0} \sum_{i}\left(x_{i+1}-x_{i}\right) \phi\left(x_{i}\right) \frac{g\left(x_{i+1}\right)-g\left(x_{i}\right)}{x_{i+1}-x_{i}}=-\int \mathrm{d} x \phi(x) \partial_{x} g(x)
\end{aligned}
$$

for any test function $\phi(x)$ and given continuous $g(x)$. Here, we invoked definition (3.1) for $F_{2}^{a}$. Note that the derivative of $g$ in the summand is evaluated at $x=x_{i}$, which is the point common to the arguments of $\phi$ and $G\left(x_{i}, x_{i+1}\right):=g\left(x_{i+1}\right)-g\left(x_{i}\right)$. Equation (4.10) will be extended to other $F_{n}^{a}$ 's in section 4.3.

4.2.2. Evaporation-condensation dynamics (ECM). In this case, we resort to macroscopic equation (2.13) by which we have

$$
\partial_{t} m-\partial_{x}^{2} \check{g}(m(x))=0,
$$

where $\check{g}(m):=g(1 / m)$. The comparison of this formula to (3.17), which is the first equation of the hierarchy for the ECM, yields

$$
\int \mathrm{d} z \mathrm{~d} y \frac{1}{a}\left[g\left(\frac{x-z}{a}\right)-g\left(\frac{y-x}{a}\right)\right] F_{3}^{a}(z, x, y) \rightarrow-\int \mathrm{d} x \phi(x) \partial_{x} \check{g}(m(x)) \quad w k .
$$

This last limit actually means that

$$
\begin{aligned}
& \int \mathrm{d} x \phi(x) \int \mathrm{d} y \mathrm{~d} z \frac{1}{a}\left[g\left(\frac{x-z}{a}\right)-g\left(\frac{y-x}{a}\right)\right] F_{3}^{a}(z, x, y) \\
& =-\sum_{i} \phi\left(x_{i+1}\right)\left[\check{g}\left(m_{i+1}\right)-\check{g}\left(m_{i}\right)\right] \rightarrow-\int \mathrm{d} x \phi(x) \partial_{x} \check{g}(m) \quad \text { as } a \rightarrow 0 .
\end{aligned}
$$

Note that the derivative of $g$ needed under summation is evaluated at $x=x_{i+1}$, the midpoint in the argument of $G\left(x_{i}, x_{i+1}, x_{i+2}\right)=\check{g}\left(m_{i+1}\right)-\check{g}\left(m_{i}\right)$.

An alternative route to obtain (4.13) is to invoke an analogue of expansion (2.1) under the summation over $i$. Aiming to expand in the step height $a$, we set $h \equiv$

\footnotetext{
${ }^{5}$ Recall the assumption that $\sup _{i}\left|x_{i+1}-x_{i}\right| \rightarrow 0$ as $a \rightarrow 0$.
} 
$h\left(x_{i+1}\right), \check{G}(h):=\check{g}\left(m_{i+1}\right)$, and, hence, $\check{G}(h+a)=\check{g}\left(m_{i}\right) \sim \check{G}(h)+a \partial_{h} \check{G}$. Thus, the left-hand side of the last line in (4.13) becomes

$$
\begin{aligned}
-\sum_{i} \phi\left(x_{i+1}\right)\left[\check{g}\left(m_{i+1}\right)-\check{g}\left(m_{i}\right)\right] & \sim \sum_{i} a \phi\left(x_{i+1}\right) \partial_{h} \check{G}\left(h\left(x_{i+1}\right)\right) \\
& \rightarrow \int \mathrm{d} h(x) \phi(x) \partial_{h} \check{g}(m \circ x(h)), \quad h:=h(x),
\end{aligned}
$$

which is identified with limit (4.13) since $\int \mathrm{d} h=\int \mathrm{d} x m(x)$.

4.2.3. Surface diffusion dynamics (SDM). By (2.23) we have the PDE

$$
\partial_{t} m+\partial_{x}^{2} \partial_{h}^{2} \check{g}(m \circ x(h)) ; \quad \partial_{h}=-m(x)^{-1} \partial_{x} .
$$

By comparison of this equation with (3.21), we find the condition

$$
\begin{gathered}
\int \mathrm{d} \vec{\ell} \frac{1}{a^{3}}\left[g\left(\frac{z-y}{a}\right)-3 g\left(\frac{y-x}{a}\right)+3 g\left(\frac{x-w}{a}\right)-g\left(\frac{w-u}{a}\right)\right] F_{5}^{a}(u, w, x, y, z) \\
\quad \rightarrow-m(x)\left[\partial_{h}^{3} \check{g}(m \circ x(h))\right] \quad w k, \quad \vec{\ell}:=(u, w, y, z) .
\end{gathered}
$$

Limit (4.16) is illustrated via the more elaborate relations

$$
\begin{aligned}
& \int \mathrm{d} x \phi(x) \int \frac{\mathrm{d} \vec{\ell}}{a^{3}}\left[g\left(\frac{z-y}{a}\right)-3 g\left(\frac{y-x}{a}\right)+3 g\left(\frac{x-w}{a}\right)-g\left(\frac{w-u}{a}\right)\right] \\
& \times F_{5}^{a}(u, w, x, y, z)=\sum_{i} \phi\left(x_{i+2}\right) \frac{1}{a^{2}}\left[\check{g}\left(m_{i+3}\right)-3 \check{g}\left(m_{i+2}\right)+3 \check{g}\left(m_{i+1}\right)-\check{g}\left(m_{i}\right)\right] \\
& \quad \rightarrow-\int \mathrm{d} x \phi(x) m(x)\left[\partial_{h}^{3} \check{g}(m \circ x(h))\right] \quad \text { as } a \rightarrow 0 .
\end{aligned}
$$

Note that the derivative of $\check{g}$ is evaluated at $x=x_{i+2}$, the midpoint in the argument of $G\left(x_{i}, x_{i+1}, x_{i+2}, x_{i+3}, x_{i+4}\right)=\check{g}\left(m_{i+3}\right)+3 \check{g}\left(m_{i+2}\right)-3 \check{g}\left(m_{i+1}\right)+\check{g}\left(m_{i}\right)$.

An alternative calculation gives more insight into limit (4.17). Invoking the height variable $h \equiv h\left(x_{i+2}\right)$, let $\check{G}(h):=\check{g}\left(m_{i+2}\right)$ and, thus, $\check{g}\left(m_{i+3}\right)=\check{G}(h-a), \check{g}\left(m_{i+1}\right)=$ $\breve{G}(h+a)$, and $\check{g}\left(m_{i}\right)=G(h+2 a)$. Hence, the summand in the second line of (4.17) is expanded in powers of $a$ yielding

$$
\begin{aligned}
& \sum_{i} \phi\left(x_{i+2}\right) \frac{1}{a^{2}}\left[\check{g}\left(m_{i+3}\right)-3 \check{g}\left(m_{i+2}\right)+3 \check{g}\left(m_{i+1}\right)-\check{g}\left(m_{i}\right)\right] \\
& \sim-\sum_{i} a \phi\left(x_{i+2}\right) \partial_{h}^{3} \check{G}\left(h\left(x_{i+2}\right)\right) \rightarrow-\int \mathrm{d} h \phi(x)\left[\partial_{h}^{3} \check{g}(m \circ x(h))\right],
\end{aligned}
$$

which readily reduces to limit (4.17). We stress the ease of taking the limit by use of the height variable $h$.

4.3. Extensions of limiting relations. We next apply Proposition 4.1 to derive the equations for the higher correlations. Such equations offer extensions of (4.9), (4.12), and (4.16) to higher $F_{n}^{a}$, which take the form of limiting rules for the passage from step sums to partial derivatives of continuous functions. These rules allude to the construction of section 6 .

Limit formula (4.3) for the step velocity is proved for the SDM in section 4.3.3 and is stated only for the other two models. In the following, $F_{n}$ denotes the limit of $F_{n}^{a}$ according to (4.2).

Copyright (C) by SIAM. Unauthorized reproduction of this article is prohibited. 
4.3.1. Toy model. Consider the hierarchy (3.15). In this case, the macroscopic equation (4.4) for $F_{n}$ becomes

$$
\partial_{t} F_{n}(\vec{y})-\sum_{j=1}^{n} \partial_{y_{j}}\left[\left(\partial_{y_{j}} g\right) \frac{F_{n}(\vec{y})}{F_{1}\left(y_{j}\right)}\right]=0
$$

where $F_{1}(x)=m(x)$ solves (4.8). Formula (4.3) holds with $v(x)=\partial_{h} g(x(h))$. The relevant vector velocity field $\vec{v}$ is

$$
\vec{v}(\vec{y}) \equiv\left(\partial_{h^{1}} g\left(y\left(h^{1}\right)\right), \ldots, \partial_{h^{n}} g\left(y\left(h^{n}\right)\right)\right), \quad h^{j}:=h\left(y_{j}\right) .
$$

We state our consequent rules for the macroscopic limit in a proposition.

Proposition 4.2. In the macroscopic limit, the functions $F_{n}^{a}$ of model (2.7) for $n \geq 2$ satisfy the relations

$$
\begin{gathered}
\frac{g\left(y_{j}\right)-g\left(y_{j+1}\right)}{a} F_{n}^{a}(\vec{y}) \rightarrow-\left(\partial_{y_{j}} g\right) \frac{F_{n}(\vec{y})}{m\left(y_{j}\right)} \quad(j=1, \ldots, n-1) \quad w k, \\
\int \mathrm{d} p \frac{1}{a}\left[g\left(y_{n}\right)-g(p)\right] F_{n+1}^{a}(\vec{y}, p) \rightarrow-\left(\partial_{y_{n}} g\right) \frac{F_{n}(\vec{y})}{m\left(y_{n}\right)} \quad w k .
\end{gathered}
$$

4.3.2. Evaporation-condensation (ECM). Next, we study hierarchy (3.18)(3.19). In this case, the fluid continuity equation (4.4) becomes

$$
\partial_{t} F_{n}(\vec{y})-\sum_{j=1}^{n} \partial_{y_{j}}\left[\partial_{y_{j}} \check{g}\left(m\left(y_{j}\right)\right) \frac{F_{n}(\vec{y})}{F_{1}\left(y_{j}\right)}\right]=0, \quad y_{j}=y\left(h^{j}\right),
$$

where $F_{1}(x)$ solves (4.11). Equation (4.3) holds with $v(x)=\partial_{h} \check{g}(m \circ x(h))$. The vector velocity field reads

$$
\vec{v}(\vec{y}) \equiv\left(\partial_{h^{1}} \check{g}\left(m \circ y\left(h^{1}\right)\right), \ldots, \partial_{h^{n}} \check{g}\left(m \circ y\left(h^{n}\right)\right)\right),
$$

where $y(h)$ is the inverse of $h(y)$.

By analogy with (4.21), we state the following proposition.

Proposition 4.3. In the macroscopic limit, the functions $F_{n}^{a}$ for model (2.10) satisfy the following relations in the weak sense:

$$
\begin{gathered}
\frac{1}{a}\left[g\left(\frac{y_{j}-y_{j-1}}{a}\right)-g\left(\frac{y_{j+1}-y_{j}}{a}\right)\right] F_{n}^{a}(\vec{y}) \rightarrow-\left[\partial_{y_{j}} \check{g}\left(m\left(y_{j}\right)\right)\right] \frac{F_{n}(\vec{y})}{m\left(y_{j}\right)}, \\
\int \mathrm{d} p \frac{1}{a}\left[g\left(\frac{y_{1}-p}{a}\right)-g\left(\frac{y_{2}-y_{1}}{a}\right)\right] F_{n+1}^{a}(p, \vec{y}) \rightarrow-\left(\partial_{y_{1}} \check{g}\right) \frac{F_{n}(\vec{y})}{m\left(y_{1}\right)}, \\
\int \mathrm{d} p \frac{1}{a}\left[g\left(\frac{y_{n}-y_{n-1}}{a}\right)-g\left(\frac{p-y_{n}}{a}\right)\right] F_{n+1}^{a}(\vec{y}, p) \rightarrow-\left(\partial_{y_{n}} \check{g}\right) \frac{F_{n}(\vec{y})}{m\left(y_{n}\right)} .
\end{gathered}
$$

4.3.3. Surface diffusion (SDM). We now turn our attention to hierarchy (3.22)-(3.25), which pertains to model (2.20).

For this model, we have the continuity law

$$
\partial_{t} F_{n}(\vec{y})+\sum_{j=1}^{n} \partial_{y_{j}}\left[\partial_{y_{j}} \partial_{h^{j}}^{2} \check{g}\left(m \circ y\left(h^{j}\right)\right) \frac{F_{n}(\vec{y})}{F_{1}\left(y_{j}\right)}\right]=0, \quad n \geq 2,
$$

Copyright $@$ by SIAM. Unauthorized reproduction of this article is prohibited. 
where $F_{1}(x)$ solves (4.15). In this case, (4.3) involves the velocity field $v(x)=$ $-\partial_{h}^{3} \check{g}(m \circ x(h))$. The associated vector velocity reads

$$
\vec{v}(\vec{y}) \equiv-\left(\partial_{h}^{3} \check{g}\left(m \circ y_{1}(h)\right), \ldots, \partial_{h^{n}}^{3} \check{g}\left(m \circ y_{n}(h)\right)\right) .
$$

First, we show (4.3) by recourse to the step velocity law (2.20) and use of the natural discretization of the height variable in increments of $a$. In view of $(2.20)$, let $h_{i+n}=h_{i}-n a$ or $x_{i+n}=x\left(h_{i}-n a\right)$, where $n=0, \pm 1, \pm 2$ and $x(h)$ is the inverse function of the height function $h(x)$ for $t$ fixed. As $a \rightarrow 0$, velocity law (2.20) is expanded as

$$
\begin{aligned}
\zeta_{i}^{a}=\frac{1}{a^{3}}[ & g\left(\frac{x\left(h_{i}-2 a\right)-x\left(h_{i}-a\right)}{a}\right)-3 g\left(\frac{x\left(h_{i}-a\right)-x\left(h_{i}\right)}{a}\right) \\
+ & \left.3 g\left(\frac{x\left(h_{i}\right)-x\left(h_{i}+a\right)}{a}\right)-g\left(\frac{x\left(h_{i}+a\right)-x\left(h_{i}+2 a\right)}{a}\right)\right] \\
=a^{-3}\{g & {\left[\partial_{h} x-\frac{1}{2} \partial_{h}^{2} x \cdot 3 a+\frac{1}{6} \partial_{h}^{3} x \cdot 7 a^{2}-\frac{1}{24} \partial_{h}^{4} x \cdot 15 a^{3}+\mathcal{O}\left(a^{4}\right)\right] } \\
& -3 g\left[\partial_{h} x-\frac{1}{2} \partial_{h}^{2} x \cdot a+\frac{1}{6} \partial_{h}^{3} x \cdot a^{2}-\frac{1}{24} \partial_{h}^{4} x \cdot a^{3}+\mathcal{O}\left(a^{4}\right)\right] \\
& +3 g\left[\partial_{h} x+\frac{1}{2} \partial_{h}^{2} x \cdot a+\frac{1}{6} \partial_{h}^{3} x \cdot a^{2}+\frac{1}{24} \partial_{h}^{4} x \cdot a^{3}+\mathcal{O}\left(a^{4}\right)\right] \\
& \left.-g\left[\partial_{h} x+\frac{1}{2} \partial_{h}^{2} x \cdot 3 a+\frac{1}{6} \partial_{h}^{3} x \cdot 7 a^{2}+\frac{1}{24} \partial_{h}^{4} x \cdot 15 a^{3}+\mathcal{O}\left(a^{4}\right)\right]\right\},
\end{aligned}
$$

where all derivatives of $x(h)$ are evaluated at $h_{i}$. By the Taylor expansion of the $g$ terms and further algebra, we obtain

$$
\begin{aligned}
\zeta_{i}^{a} & =-\left[g^{\prime}\left(\partial_{h} x\right) \partial_{h}^{4} x+g^{\prime \prime}\left(\partial_{h} x\right) \cdot 3 \partial_{h}^{2} x \partial_{h}^{3} x+g^{\prime \prime \prime}\left(\partial_{h} x\right)\left(\partial_{h}^{2} x\right)^{3}\right]+\mathcal{O}(a) \\
& =-\partial_{h}^{3}\left[g\left(\partial_{h} x\right)\right]+\mathcal{O}(a),
\end{aligned}
$$

where the term $\mathcal{O}(a)$ can be clearly estimated uniformly in $i$ in terms of higher-order derivatives. This assertion concludes the derivation of (4.3).

By analogy with (4.21) and (4.24), we state the following proposition.

PROPOSITION 4.4. In the macroscopic limit, the functions $F_{n}^{a}$ for model (2.20) in correspondence to (3.22)-(3.25), satisfy the following relations:

$$
\begin{aligned}
& \int \mathrm{d} z \mathrm{~d} w \mathrm{~d} u \frac{1}{a^{3}}\left[g\left(\frac{z-y}{a}\right)-3 g\left(\frac{y-x}{a}\right)+3 g\left(\frac{x-w}{a}\right)-g\left(\frac{w-u}{a}\right)\right] F_{5}^{a}(u, w, x, y, z) \\
& \text { (4.29a) } \rightarrow\left[\partial_{x} \partial_{h}^{2} \check{g}(m \circ x(h))\right] \frac{F_{2}(x, y)}{F_{1}(x)} \quad w k, \\
& \int \mathrm{d} z \mathrm{~d} w \mathrm{~d} u \frac{1}{a^{3}}\left[g\left(\frac{u-z}{a}\right)-3 g\left(\frac{z-y}{a}\right)+3 g\left(\frac{y-x}{a}\right)-g\left(\frac{x-w}{a}\right)\right] F_{5}^{a}(w, x, y, z, u) \\
& \rightarrow\left[\partial_{y} \partial_{h}^{2} \check{g}(m \circ y(h))\right] \frac{F_{2}(x, y)}{F_{1}(y)} \quad w k,
\end{aligned}
$$

Copyright $\odot$ by SIAM. Unauthorized reproduction of this article is prohibited. 


$$
\begin{aligned}
& \int \mathrm{d} u \mathrm{~d} w \frac{1}{a^{3}}\left[g\left(\frac{u-z}{a}\right)-3 g\left(\frac{z-y}{a}\right)+3 g\left(\frac{y-x}{a}\right)-g\left(\frac{x-w}{a}\right)\right] F_{5}^{a}(w, x, y, z, u) \\
& (4.29 \mathrm{c}) \rightarrow\left[\partial_{y} \partial_{h}^{2} \check{g}(m \circ y(h))\right] \frac{F_{3}(x, y)}{F_{1}(y)} \quad w k, \\
& \int \mathrm{d} p \mathrm{~d} s \frac{1}{a^{3}}\left[g\left(\frac{y_{3}-y_{2}}{a}\right)-3 g\left(\frac{y_{2}-y_{1}}{a}\right)+3 g\left(\frac{y_{1}-s}{a}\right)-g\left(\frac{s-p}{a}\right)\right] F_{n+2}^{a}(p, s, \vec{y}) \\
& (4.29 \mathrm{~d}) \rightarrow\left[\partial_{y_{1}} \partial_{h^{1}}^{2} \check{g}\left(m \circ y\left(h^{1}\right)\right)\right] \frac{F_{n}(\vec{y})}{F_{1}\left(y_{1}\right)} \quad w k, \quad n \geq 3, \\
& \int \mathrm{d} s \mathrm{~d} p \frac{1}{a^{3}}\left[g\left(\frac{s-p}{a}\right)-3 g\left(\frac{p-y_{n}}{a}\right)+3 g\left(\frac{y_{n}-y_{n-1}}{a}\right)-g\left(\frac{y_{n-1}-y_{n-2}}{a}\right)\right] \\
& \times F_{n+2}^{a}(\vec{y}, p, s) \rightarrow\left[\partial_{y_{n}} \partial_{h^{n}}^{2} \check{g}\left(m \circ y\left(h^{n}\right)\right)\right] \frac{F_{n}(\vec{y})}{F_{1}\left(y_{n}\right)} \quad w k, \quad n \geq 3, \\
& \int \mathrm{d} s \frac{1}{a^{3}}\left[g\left(\frac{y_{4}-y_{3}}{a}\right)-3 g\left(\frac{y_{3}-y_{2}}{a}\right)+3 g\left(\frac{y_{2}-y_{1}}{a}\right)-g\left(\frac{y_{1}-s}{a}\right)\right] F_{n+1}^{a}(s, \vec{y}) \\
& \rightarrow\left[\partial_{y_{2}} \partial_{h^{2}}^{2} \check{g}\left(m \circ y\left(h^{2}\right)\right)\right] \frac{F_{n}(\vec{y})}{F_{1}\left(y_{2}\right)} \quad w k, \quad n \geq 4, \\
& \int \mathrm{d} s \frac{1}{a^{3}}\left[g\left(\frac{s-y_{n}}{a}\right)-3 g\left(\frac{y_{n}-y_{n-1}}{a}\right)+3 g\left(\frac{y_{n-1}-y_{n-2}}{a}\right)-g\left(\frac{y_{n-2}-y_{n-3}}{a}\right)\right] \\
& \times F_{n+1}^{a}(\vec{y}, s) \rightarrow\left[\partial_{y_{n-1}} \partial_{h^{n-1}}^{2} \check{g}\left(m \circ y\left(h^{n-1}\right)\right)\right] \frac{F_{n}(\vec{y})}{F_{1}\left(y_{n-1}\right)} \quad w k, \quad n \geq 4, \\
& \frac{1}{a^{3}}\left[g\left(\frac{y_{j+2}-y_{j+1}}{a}\right)-3 g\left(\frac{y_{j+1}-y_{j}}{a}\right)+3 g\left(\frac{y_{j}-y_{j-1}}{a}\right)-g\left(\frac{y_{j-1}-y_{j-2}}{a}\right)\right] F_{n}^{a}(\vec{y}) \\
& \rightarrow\left[\partial_{y_{j}} \partial_{h^{j}}^{2} \check{g}\left(m \circ y\left(h^{j}\right)\right)\right] \frac{F_{n}(\vec{y})}{F_{1}\left(y_{j}\right)} \quad w k, \quad j=3, \ldots, n-2 ; n \geq 5,
\end{aligned}
$$

where $h^{j}=h\left(y_{j}\right)$.

4.4. Remark on the use of step height. In the derivations of the previous subsections, the step position $x_{i}$ is expressed as a monotone function of the surface height $h$. This formulation is enabled by the following assumptions: (i) steps do not cross and, thus, their ordering is preserved by the flow; and (ii) the step train is monotonic. The use of $h$ as an independent variable has offered a convenience in carrying out the macroscopic limit.

To elaborate on this point, consider a system of particles $\left\{x_{i}\right\}(i=1, \ldots N)$ with the following properties: (i) their ordering is preserved with time; (ii) $\min _{i}\left|x_{i+1}-x_{i}\right|=$ $\mathcal{O}(\delta)$; and (iii) $\max _{i}\left|x_{i+1}-x_{i}\right| / \min _{i}\left|x_{i+1}-x_{i}\right|=\mathcal{O}(1)$ as $\delta \rightarrow 0$. Then, we can define a function $X(s, t)$ such that $X(i \delta, t)=x_{i}(t)$ for fixed $t$. The variable $s$ can be viewed as an apparent height with $\delta$ being the step height. In the macroscopic limit, $\delta \rightarrow 0$ and $N \rightarrow \infty$ with $N \delta=\mathcal{O}(1)$. The analysis proceeds along the same lines as before.

5. Solving the macroscopic equations for $\boldsymbol{F}_{\boldsymbol{n}}$. In this section we solve the macroscopic transport equations (4.19), (4.22), and (4.25) for $F_{n}(n \geq 2)$ in a unified framework. Each of these equations reads as the continuity equation (4.4) with the velocity field

$$
\vec{v}(\vec{y})=\left(v_{1}, \ldots, v_{n}\right)=\nabla_{\vec{h}} \widetilde{\mathcal{G}}(\vec{h}) ; \quad \widetilde{\mathcal{G}}:=\sum_{j=1}^{n} \mathcal{G}\left(y\left(h^{j}\right)\right)
$$

Copyright (C) by SIAM. Unauthorized reproduction of this article is prohibited. 
where $v_{j}=v\left(y_{j}\right)=\partial_{t} h /\left|\partial_{y_{j}} h\right|, y(h)$ is the inverse of $h(y), \nabla_{\vec{h}}:=\left(\partial_{h^{1}}, \ldots, \partial_{h^{n}}\right)$, and

$$
\mathcal{G}(x)=\left\{\begin{array}{lr}
g(x) & \mathrm{TM}, \\
\check{g}(m(x)) & \mathrm{ECM}, \\
-\partial_{h}^{2} \check{g}(m \circ x(h)) & \mathrm{SDM} .
\end{array}\right.
$$

Recall that the singlet density $F_{1}(x)$ satisfies PDE (4.8), (4.11), or (4.15). These PDEs are recast to the form

$$
\partial_{t} F_{1}(x)-\partial_{x}^{2} \mathcal{G}(x)=0,
$$

which stems from the motion law $v(x)=\partial_{t} h /\left|\partial_{x} h\right|$ or

$$
\partial_{t} h(x)+\partial_{x} \mathcal{G}(x)=0 .
$$

Our task is to solve (4.4) under (5.1)-(5.4). We observe that (4.4) has the particular solution

$$
F_{n}(\vec{y})=F_{n-1}\left(\vec{y}^{n}\right) F_{1}\left(y_{n}\right), \quad n \geq 2,
$$

where $F_{1}(x)$ solves (5.3). Equation (5.5) of course cannot be applied to the original, finite- $a$ hierarchies. However, its simple form motivates the following question: what is the general solution of (4.4)?

Let us consider first the case with $n=2$, i.e., study the pair correlation function $F_{2}$. In view of (5.5), we introduce the decomposition ${ }^{6}$

$$
F_{2}(x, y, t)=F_{1}(x, t) F_{1}(y, t) \Lambda(x, y, t) .
$$

Note that $\Lambda$ describes the degree of decorrelation of the solution (with the value $\Lambda=1$ corresponding to independence).

Introducing (5.6) into (4.4) with (5.1), it follows that $\Lambda(x, y, t)$ satisfies

$$
\partial_{t} \Lambda+v(x, t) \partial_{x} \Lambda+v(y, t) \partial_{y} \Lambda=0, \quad v(x, t)=-\partial_{t} h / \partial_{x} h .
$$

Equation (5.7) has as solution

$$
\Lambda(x, y, t)=C(h(x, t), h(y, t))
$$

where $C$ is arbitrary and is computed from $\Lambda(x, y, 0)$, i.e., the degree of decorrelation of the initial data. This solution stems from noticing that $\vec{v}=(v(x, t), v(y, t))=$ $\left(\partial_{t} h /\left|\partial_{x} h\right|, \partial_{t} h /\left|\partial_{y} h\right|\right)$ defines the characteristics (or particle paths) for PDE (5.7), along which $\Lambda=$ constant. Thus, we reach the following result.

Proposition 5.1. The macroscopic limit of the pair correlation function $F_{2}^{a}$ has the form

$$
F_{2}(x, y, t)=F_{1}(x, t) F_{1}(y, t) C(h(x, t), h(y, t)),
$$

where $F_{1}(x, t)$ satisfies (5.3), $h(x, t)$ satisfies (5.4), and $C\left(h^{x}, h^{y}\right)$ is computed in terms of the initial data $F_{2}(x, y, 0)$ and $F_{1}(x, 0)$.

This result can be extended to other $F_{n}$ 's $(n \geq 3)$. The general case can be stated as follows.

\footnotetext{
${ }^{6}$ At this stage, we indicate the explicit time dependence of dynamic variables.
} 
Proposition 5.2. In the macroscopic limit, the functions $F_{n}^{a}(\vec{y}, t)$ become

$$
F_{n}(\vec{y}, t)=: F_{n}\left(\vec{y}, t ;\left[C_{n}\right]\right)=F_{n-1}\left(\vec{y}^{n}, t\right) F_{1}\left(y_{n}, t\right) C_{n}(\vec{h}), \quad n \geq 2,
$$

where $F_{1}(x, t)$ satisfies (5.3), $h(x, t)$ satisfies (5.4), and $C_{n}$ is a function of $\vec{h}=$ $\left(h\left(y_{1}, t\right), \ldots, h\left(y_{n}, t\right)\right)$, subject to (4.1), and is computed in terms of the initial data $F_{n}(\vec{y}, 0), n \geq 1$.

A particular yet physically appealing case arises when $F_{n}^{a}$ depend on differences of the height variables. This case is illustrated by the pair correlation function $F_{2}(x, y, t)$ as follows.

Corollary 5.3. A family of solutions for $F_{2}(x, y, t)$ is described by

$$
F_{2}(x, y, t)=F_{1}(x, t) F_{1}(y, t) \mathcal{C}(h(x, t)-h(y, t)),
$$

where $\mathcal{C}(u)$ is (reasonably) arbitrary, $F_{1}$ satisfies (5.3), and $h$ solves (5.4).

Similar families of solutions with reduced variables can be written for $F_{n}, n \geq 3$.

6. A construction of $\boldsymbol{F}_{\boldsymbol{n}}^{a}, \mathbf{0}<\boldsymbol{a} \ll \mathbf{1}$. In the previous section we treated the limits of $F_{n}^{a}$ as classical, sufficiently smooth solutions of transport equations. This approach is reasonable since, in the macroscopic limit, it is meaningful that steps coalesce to form a continuous surface. However, $F_{n}^{a}$ are originally defined as measures by (3.1) for which, strictly speaking, our procedure for solutions is not valid. In this section we restrict attention to small but finite $a$ and construct particular $F_{n}^{a}$ that are regularizations of measures. Such $F_{n}^{a}$ become singular in the limit $a \rightarrow 0$ and, hence, exhibit behavior different from that based on the general PDE solutions found above. Our construction encompasses all three models reflected in (5.2) and has been inspired by the limiting relations of sections 4.2 and 4.3 .

Accordingly, with recourse to Proposition 5.2, the key idea is to introduce the dependence on height variables through appropriate mollifiers whose features scale with $a$; see Propositions $6.1,6.2$, and 6.3. We start by specifying the function $C_{n}$ for the general solution (5.10) via the formula ${ }^{7} C_{n}=a^{-1} C^{a}\left(\vec{\xi}^{n}\right)$, where $C^{a}$ represent a family of mollifiers in $\mathbb{R}^{n-1}$, i.e.,

$$
\begin{aligned}
& C^{a} \geq 0, \quad \int_{\mathbb{R}^{n-1}} \mathrm{~d} \vec{\xi}^{n} C^{a}=1 \quad\left(\vec{\xi}^{n}:=\left(\xi_{1}, \ldots, \xi_{n-1}\right)\right), \\
& \lim _{a \rightarrow 0} \int_{\left|\vec{\xi}^{n}\right|>\delta} \mathrm{d} \vec{\xi}^{n} C^{a}\left(\vec{\xi}^{n}\right)=0 \quad \text { for all } \delta=\mathcal{O}(1)>0 ;
\end{aligned}
$$

in addition, in compliance with (4.1), $C^{a}$ satisfy

$$
C^{a}\left(\vec{\xi}^{n}\right) \equiv 0 \quad \text { if } \xi_{j}<0
$$

where $\vec{\xi}^{n}:=\left(\left(h^{1}-h^{2}\right) / a-1, \ldots,\left(h^{n-1}-h^{n}\right) / a-1\right)$. On the basis of (5.10), we thereby define ${ }^{8}$

$$
F_{n}^{a}(\vec{y}):=F_{n}\left(\vec{y} ;\left[C^{a}\right]\right)
$$

and show that there is a choice for $C^{a}$ consistent with (4.21), (4.24), and (4.29); in this way, the hierarchies of section 3 are approximately solved, being reduced to

\footnotetext{
${ }^{7}$ To avoid confusion, we suppress the (trivial) dependence of the arbitrary function $C$ on $n$.

${ }^{8}$ We return to our notational economy of suppressing time dependence; recall that $h(x)$ and all $F_{n}^{a}(\vec{y})$ depend explicitly on $t$.
} 
their assumed macroscopic limits. The consistency of this construction with plausible initial data for $F_{n}^{a}$ is not studied here but needs to be explored.

In the remainder of this section, we study a particular, simple class of $C^{a}$, namely, $C^{a}=C^{a}\left(\xi_{n-1}\right)$. This choice is made for the sake of simplicity.

6.1. Pair correlation. For the sake of clarity, let us consider first the pair correlation function $F_{2}^{a}(x, y)$ which enters a nonlocal term in the hierarchy of the toy model; cf. (3.14). We apply the ansatz

$$
F_{2}^{a}(x, y) \sim \frac{1}{a} F_{1}(x) F_{1}(y) \nu_{1}^{a}\left(\frac{h^{x}-h^{y}}{a}-1\right), \quad h^{x}=h(x),
$$

where we have taken $C^{a}(\xi)=: \nu_{1}^{a}(\xi)$. By $(6.1)$, we require that

$$
\begin{gathered}
\nu_{1}^{a}(\xi) \geq 0, \quad \int_{0}^{+\infty} \mathrm{d} \xi \nu_{1}^{a}(\xi)=1, \quad \lim _{a \rightarrow 0} \int_{\delta}^{+\infty} \mathrm{d} \xi \nu_{1}^{a}(\xi)=0 \\
\nu_{1}^{a}(\xi) \equiv 0 \quad \text { for } \xi<0
\end{gathered}
$$

For example, an admissible choice is the normalized Gaussian

$$
\nu_{1}^{a}(\xi)=\sqrt{\frac{2}{\pi a}} H(\xi) e^{-\xi^{2} /(2 a)}
$$

where $H(\xi)$ is the Heaviside function; i.e., $H(\xi)=0$ if $\xi<0$ and $H(\xi)=1$ if $\xi>0$. Note that (6.5) approaches a "one-sided" delta function as $a \rightarrow 0$.

It follows that (3.3) is automatically satisfied by the normalization of $\nu_{1}^{a}$. Another constraint should account for (4.9) along with (4.21) of Proposition 4.2. Since $\nu_{1}^{a}(\xi)$ is rapidly decreasing away from $\xi=0$, we reduce the nonlocal term of (4.9) to the integral

$$
\begin{aligned}
\int \mathrm{d} y a^{-1}[g(x)-g(y)] F_{2}^{a}(x, y) & \sim m(x) \int \mathrm{d} \xi a^{-1}[g(x(h))-g(x(h-a(1+\xi)))] \nu_{1}^{a}(\xi) \\
& \sim m(x)\left[\partial_{h} g(x(h))\right] \int \mathrm{d} \xi(1+\xi) \nu_{1}^{a}(\xi) \sim-\partial_{x} g(x),
\end{aligned}
$$

where we set $1+\xi \sim 1$ under the integral sign because the $\xi$-region (in the neighborhood of $\xi=0$ ) contributing to integration is vanishingly small as $a \rightarrow 0 ; x(h)$ denotes the inverse of $h=h(x)$.

Without further ado, we arrive at the following statement.

Proposition 6.1. For sufficiently small step height (a), a family of pair correlation functions $F_{2}^{a}(x, y)$ is given by asymptotic formula (6.3) where $F_{1}(x)=m(x)$ satisfies (5.3) and $\nu_{1}^{a}(\xi)$ is a family of mollifiers subject to conditions (6.1).

6.2. Triplet correlation. The function $F_{3}^{a}$ is treated in the same spirit as $F_{2}^{a}$. Nonlocal terms containing $F_{3}^{a}$ enter (3.15) for the TM and part of hierarchy (3.17)(3.19) for the ECM. We study $F_{3}^{a}$ separately here since it provides an example of approximations needed in the context of the ECM.

By extending (6.3), we have the following proposition.

Proposition 6.2. For sufficiently small step height (a), a family of triplet correlation functions is given by the asymptotic formula

$$
F_{3}^{a}(x, y, z) \sim \frac{1}{a} F_{2}^{a}(x, y) F_{1}(z) \nu_{2}^{a}\left(\frac{h^{y}-h^{z}}{a}-1\right),
$$

Copyright $@$ by SIAM. Unauthorized reproduction of this article is prohibited. 
where $F_{1}(x)$ satisfies (5.3), $h^{w}=h(w)$ ( $\left.w=y, z\right)$ obeys (5.4), $F_{2}^{a}$ is provided by (6.3), and $\nu_{2}^{a}(\xi)$ is subject to conditions (6.4).

Evidently, (6.3) is consistent with (3.3). Further, by comparison to (4.12) and Propositions 4.2 and 4.3 , we can verify that (6.7) is consistent with the hierarchies of the TM and ECM near their macroscopic limits. For example, the nonlocal term in (3.17) for the ECM becomes

$$
\begin{aligned}
F_{1}(x) & \int \mathrm{d} \xi_{1} \mathrm{~d} \xi_{2} \frac{1}{a}\left[g\left(\frac{x(h)-x\left(h+a\left(1+\xi_{1}\right)\right)}{a}\right)-g\left(\frac{x\left(h-\left(1+\xi_{2}\right) a\right)-x(h)}{a}\right)\right] \\
& \times \nu_{1}^{a}\left(\xi_{1}\right) \nu_{2}^{a}\left(\xi_{2}\right) \\
& \sim F_{1}(x) \frac{1}{a}\left[g\left(\frac{x(h)-x(h+a)}{a}\right)-g\left(\frac{x(h-a)-x(h)}{a}\right)\right] \int \mathrm{d} \vec{\xi} \nu_{1}^{a}\left(\xi_{1}\right) \nu_{2}^{a}\left(\xi_{2}\right) \\
(6.8) & \sim F_{1}(x)\left[\partial_{h} \check{g}(m \circ x(h))\right], \quad \vec{\xi}=\left(\xi_{1}, \xi_{2}\right),
\end{aligned}
$$

which is in agreement with (4.12). Note that carrying out the asymptotics of the requisite integral is facilitated by use of the height variable. Similarly, we can show that (6.7) is consistent with (4.21) and (4.24).

6.3. Higher correlations. We describe our particular construction for $F_{n}^{a}$ in the form of a proposition.

Proposition 6.3. A family of n-step correlation functions $F_{n}^{a}$ can be constructed asymptotically according to

$$
F_{n}^{a}(\vec{y}) \sim \frac{1}{a} F_{n-1}^{a}\left(\vec{y}^{n-1}\right) F_{1}\left(y_{n}\right) \nu_{n-1}^{a}\left(\frac{h^{n-1}-h^{n}}{a}-1\right), \quad n \geq 2,
$$

where $F_{1}(x)$ satisfies (5.3), $h^{j}=h\left(y_{j}\right)$ obey $(5.4)$, and $\nu_{j}^{a}(\xi)$ are subject to (6.1).

It can be verified that ansatz (6.9) meets the constraints for $F_{n}^{a}$, i.e., the integral condition (3.3), the macroscopic limits (4.10), (4.12), and (4.16), along with Propositions 4.2, 4.3, and 4.4. We illustrate the use of (6.9) for $n=5$ in the context of the $\mathrm{SDM}$, particularly for the nonlocal term in $(3.21)$ for $F_{1}^{a}(x)$. This term becomes

$$
\begin{aligned}
& \int \mathrm{d} u \mathrm{~d} w \mathrm{~d} y \mathrm{~d} z \frac{1}{a^{3}}\left[g\left(\frac{z-y}{a}\right)-3 g\left(\frac{y-x}{a}\right)+3 g\left(\frac{x-w}{a}\right)-g\left(\frac{w-u}{a}\right)\right] F_{5}^{a} \\
\sim & F_{1}(x) \int \mathrm{d} \vec{\xi} \frac{1}{a^{3}}\left[g\left(\frac{x\left(h-a\left(2+\xi_{2}+\xi_{3}\right)\right)-x\left(h-a\left(1+\xi_{3}\right)\right)}{a}\right)\right. \\
& -3 g\left(\frac{x\left(h-a\left(1+\xi_{4}\right)\right)-x(h)}{a}\right)+3 g\left(\frac{x(h)-x\left(h+a\left(1+\xi_{2}\right)\right)}{a}\right) \\
& \left.-g\left(\frac{x\left(h+a\left(1+\xi_{2}\right)\right)-x\left(h+a\left(2+\xi_{1}+\xi_{2}\right)\right)}{a}\right)\right] \prod_{j=1}^{4} \nu_{j}^{a}\left(\xi_{j}\right) \\
\sim & F_{1}(x) \frac{1}{a^{3}}\left[g\left(\frac{x(h-2 a)-x(h-a)}{a}\right)-3 g\left(\frac{x(h-a)-x(h)}{a}\right)\right. \\
& \left.+3 g\left(\frac{x(h)-x(h+a)}{a}\right)-g\left(\frac{x(h+a)-x(h+2 a)}{a}\right)\right] \int \mathrm{d} \vec{\xi} \prod_{j=1}^{4} \nu_{j}^{a}\left(\xi_{j}\right) \\
& \sim-F_{1}(x)\left[\partial_{h}^{3} \check{g}(m \circ x(h))\right], \quad \vec{\xi}=\left(\xi_{1}, \ldots, \xi_{4}\right) .
\end{aligned}
$$

Copyright (C) by SIAM. Unauthorized reproduction of this article is prohibited. 
7. Conclusion. The motion of steps on crystal surfaces in $1+1$ dimensions was studied from the Eulerian perspective of kinetic hierarchies. The governing, discrete equations describe the evolution of Lagrangian coordinates, the step positions. In contrast, the kinetic hierarchies provide a description of step correlation functions $F_{n}^{a}$. We studied the hierarchies near the macroscopic limit, when the step height approaches zero and the stepped surface resembles a continuous medium. In particular, we derived limiting relations for $F_{n}^{a}$ by which nonlocal couplings in evolution laws reduce to local terms. The hierarchies simplify to simple transport equations for the limits $F_{n}, n \geq 2$. Each $F_{n}$ is slaved to the positive surface slope $F_{1}(x)$, which satisfies known PDEs of epitaxial relaxation.

Because of the specific form of the velocity in the continuity equations for $F_{n}$, we were able to determine general solutions depending on height variables. The propagation of chaos is a special case of these solutions.

We also studied $F_{n}^{a}$ for finite but small $a$ on the basis of their definition as measurevalued functions. By regularizing measures in an appropriate sense, we constructed a particular family of $F_{n}^{a}$ when $a \ll 1$ by the use of mollifiers whose features scale with $a$. In this context, $F_{n}^{a}$ become singular as $a \rightarrow 0$.

The kinetic description of this article can be easily extended to the case with material deposition from above. Deposition is included trivially at the nanoscale: a constant source term is added in the diffusion equation for adatoms [29]. Consequently, the step velocity law is still of the form (2.2) and the BBGKY-type hierarchy (3.8)-(3.12) remains valid.

Our approach has limitations, which point to unresolved questions. For example, it does not treat surface profiles with corrugations, where the height ceases to be monotonic. Furthermore, we study correlation functions for step positions instead of the more experimentally accessible terrace widths [8].

Our work describes the derivation of macroscopic equations for the correlation functions of a particle system under the assumption that in the macroscopic limit the particles organize themselves on a smooth surface. In this sense, our work is formal. A rigorous study of these derivations would require uniform a priori estimates (in $N$ ) for the step density and higher-order correlations and lies beyond the scope of this article.

Our approach is fully deterministic. A tractable and more physically appealing case is to apply initial data with known statistics and study the evolution of joint probability densities for step positions under deterministic step velocity laws. Even more interesting is the application of noise to the nanoscale kinetic processes (and the resulting step velocities) by which steps fluctuate in $2+1$ dimensions. Inclusion of stochastic effects in the discrete schemes is a viable direction of future research.

Acknowledgments. We are indebted to Govind Menon for extensive and stimulating discussions. We also wish to thank Theodore L. Einstein, David Levermore, Olivier Pierre-Louis, John D. Weeks, and Ellen D. Williams for valuable conversations. One of us (D. Margetis) appreciates the support of the Maryland NanoCenter.

\section{REFERENCES}

[1] A. N. Benson, H. L. Richards, and T. L. Einstein, Step-position distributions and the pairwise Einstein model for steps on crystal surfaces, Phys. Rev. B, 73 (2006), 115429.

[2] W. K. Burton, N. Cabrera, and F. C. Frank, The growth of crystals and the equilibrium structure of their surfaces, Philos. Trans. R. Soc. London Ser. A Math. Phys. Eng. Sci., 243 (1951), pp. 299-358. 
[3] R. E. Caflisch, W. E, M. F. Gyure, B. Merriman, and C. Ratsch, Kinetic model for a step edge in epitaxial growth, Phys. Rev. E, 59 (1999), pp. 6879-6887.

[4] C. Cercignani, R. Illner, and M. Pulvirenti, The Mathematical Theory of Dilute Gases, Springer, Berlin, 1994.

[5] A. Chame, S. Rousset, H. P. Bonzel, and J. Villain, Slow dynamics of stepped surfaces, Bulgarian Chem. Commun., 29 (1996/97), pp. 398-434.

[6] N. Combe, P. Jensen, And A. Pimpinelli, Changing shapes in the nanoworld, Phys. Rev. Lett., 85 (2000), pp. 110-113.

[7] C. Duport, P. Politi, and J. Villain, Growth instabilities induced by elasticity in a vicinal surface, J. Phys. I, 5 (1995), pp. 1317-1350.

[8] T. L. Einstein, Applications of ideas from random matrix theory to step distributions on "misoriented" surfaces, Ann. Henri Poincaré, 4 (2003), pp. S811-S824.

[9] J. W. Evans, P. A. Thiel, And M. C. Bartelt, Morphological evolution during epitaxial thin film growth: Formation of $2 D$ islands and $3 D$ mounds, Surf. Sci. Rep., 61 (2006), pp. $1-128$.

[10] P.-W. Fok, R. R. Rosales, And D. Margetis, Unification of step bunching phenomena on vicinal surfaces, Phys. Rev. B, 76 (2007), 033408.

[11] E. E. Gruber And W. W. MulLins, On the theory of anisotropy of crystalline surface tension, J. Phys. Chem. Solids, 28 (1967), pp. 875-887.

[12] K. Huang, Statistical Mechanics, Wiley, New York, 1987.

[13] N. Israeli and D. Kandel, Profile of a decaying crystalline cone, Phys. Rev. B, 60 (1999), pp. 5946-5962.

[14] N. Israeli and D. Kandel, Decay of one-dimensional surface modulations, Phys. Rev. B, 62 (2000), pp. 13707-13717.

[15] C. Jayaprakash, C. Rottman, and W. F. SaAm, Simple model for crystal shapes: Step-step interactions and facet edges, Phys. Rev. B, 30 (1984), pp. 6549-6554.

[16] H.-C. Jeong and E. D. Williams, Steps on surfaces: Experiments and theory, Surf. Sci. Rep., 34 (1999), pp. 171-294.

[17] F. Liu, J. Tersoff, and M. G. Lagally, Self-organization of steps in growth of strained films on vicinal substrates, Phys. Rev. Lett., 80 (1998), pp. 1268-1271.

[18] V. I. Marchenko And A. Y. Parshin, Elastic properties of crystal surfaces, Soviet Phys. JETP, 52 (1980), pp. 129-131.

[19] D. Margetis, Unified continuum approach to crystal surface morphological relaxation, Phys. Rev. B, 76 (2007), 193403.

[20] D. Margetis, M. J. Aziz, And H. A. Stone, Continuum approach to self-similarity and scaling in morphological relaxation of a crystal with a facet, Phys. Rev. B, 71 (2005), 165432 .

[21] D. Margetis and R. E. Caflisch, Anisotropic step stiffness from a kinetic model of epitaxial growth, Multiscale Model. Simul., 7 (2008), pp. 242-273.

[22] D. Margetis, P.-W. Fok, M. J. Aziz, and H. A. Stone, Continuum theory of nanostructure decay via a microscale condition, Phys. Rev. Lett., 97 (2006), 096102.

[23] D. Margetis and R. V. Kohn, Continuum theory of interacting steps on crystal surfaces in $2+1$ dimensions, Multiscale Model. Simul., 5 (2006), pp. 729-758.

[24] T. Michely and J. Krug, Islands, Mounds and Atoms: Patterns and Processes in Crystal Growth Far from Equilibrium, Springer Ser. Surf. Sci., Springer, Berlin, 2004.

[25] R. Najafabadi and D. J. Srolovitz, Elastic step interactions on vicinal surfaces of fcc metals, Surf. Sci., 317 (1994), pp. 221-234.

[26] P. NoziÉRes, On the motion of steps on a vicinal surface, J. Phys. (France), 48 (1987), pp. $1605-1608$.

[27] J. O'Connell, F. Sullivan, D. Libes, E. Orlandini, M. C. Tesi, A. L. Siella, and T. L. EinsteIn, Self-avoiding random surfaces: Monte Carlo study using oct-tree datastructure, J. Phys. A, 24 (1991), pp. 4619-4635.

[28] M. Ozdemir AND A. ZANGwill, Morphological equilibration of a corrugated crystalline surface, Phys. Rev. B, 42 (1990), pp. 5013-5024.

[29] P. Politi And J. Villain, Ehrlich-Schwoebel instability in molecular-beam epitaxy: A minimal model, Phys. Rev. B, 54 (1996), pp. 5114-5129.

[30] M. Pulvirenti, Kinetic limits for stochastic particle systems, in Probabilistic Models for Nonlinear Partial Differential Equations, Montecatini Terme, Italy, Lecture Notes Math., D. Talay and L. Tubaro, eds., Springer, Berlin, 1995, pp. 96-126.

[31] A. Rettori and J. Villain, Flattening of grooves on a crystal surface: A method of investigation of surface roughness, J. Phys. (France), 49 (1988), pp. 257-267.

[32] J. M. Rickman and D. J. Srolovitz, Defect interactions on solid surfaces, Surf. Sci., 284 (1993), pp. 211-221.

Copyright $@$ by SIAM. Unauthorized reproduction of this article is prohibited. 
[33] V. B. Shenoy And L. B. Freund, A continuum description of the energetics and evolution of stepped surfaces in strained nanostructures, J. Mech. Phys. Solids, 50 (2002), pp. 18171841.

[34] H. Sponn, Large Scale Dynamics of Interacting Particles, Springer, New York, 1991.

[35] H. Spohn, Surface dynamics below the roughening transition, J. Phys. I (France), 3 (1993), pp. 69-81.

[36] H. A. Stone, M. J. Aziz, And D. Margetis, Grooving of a grain boundary by evaporationcondensation below the roughening transition, J. Appl. Phys., 97 (2005), 113535.

Copyright (c) by SIAM. Unauthorized reproduction of this article is prohibited. 